\title{
Rho meson condensation at finite isospin chemical potential in a holographic model for QCD
}

\author{
Ofer Aharony ${ }^{1}$, Kasper Peeters ${ }^{2}$, Jacob Sonnenschein ${ }^{3,4}$ and Marija Zamaklar ${ }^{5}$ \\ ${ }^{1}$ Department of Particle Physics, Weizmann Institute of Science, Rehovot 76100, Israel. \\ ${ }^{2}$ Institute for Theoretical Physics, Utrecht University, P.O. Box 80.195, 3508 TD \\ Utrecht, The Netherlands. \\ ${ }^{3}$ School of Physics and Astronomy, The Raymond and Beverly Sackler Faculty of Exact \\ Sciences, Tel Aviv University, Ramat Aviv, 69978, Israel. \\ ${ }^{4}$ Albert Einstein Minerva Center, Weizmann Institute of Science, Rehovot 76100, Israel. \\ ${ }^{5}$ Department of Mathematical Sciences, Durham University, South Road, Durham DH1 \\ 3LE, United Kingdom.
}

\begin{tabular}{|l|}
\hline ofer.aharony@weizmann.ac.il \\
\hline kasper.peeters@aei.mpg.de \\
\hline cobi@post.tau.ac.il \\
\hline marija.zamaklar@durham.ac.uk \\
\hline
\end{tabular}

Abstract: We analyze the effect of an isospin chemical potential $\mu_{I}$ in the Sakai-Sugimoto model, which is the string dual of a confining gauge theory related to large $N_{c}$ QCD, at temperatures below the chiral symmetry restoration temperature. For small chemical potentials we show that the results agree with expectations from the low-energy chiral Lagrangian, and the charged pion condenses. When the chemical potential reaches a critical value $\mu_{I}=\mu_{\text {crit }} \simeq 1.7 m_{\rho}$, the lowest vector meson (the "rho meson") becomes massless, and it condenses (in addition to the pion condensate) for $\mu_{I}>\mu_{\text {crit }}$. This spontaneously breaks the rotational symmetry, as well as a residual $U(1)$ flavor symmetry. We numerically construct the resulting new ground state for $\mu_{I}>\mu_{\text {crit }}$. 


\section{Contents}

1. Introduction and summary of results 1

2. The configurations with no chemical potential 5

2.1 Review of the phases of the Sakai-Sugimoto model 5

2.2 The chiral symmetry and the moduli space 7

3. Isotropic solutions with flavor chemical potentials 9

3.1 Flavor chemical potentials in the Sakai-Sugimoto model

3.2 Chemical potentials in the chiral Lagrangian 11

3.3 Isotropic solutions for small chemical potentials 13

3.4 Isotropic solutions for large flavor chemical potentials 17

4. Instability of isotropic solutions and rho meson condensation 20

4.1 The fluctuation equations 20

4.2 Transverse vectors and scalars 22

4.3 Pions and longitudinal vectors 24

4.4 Vector meson condensation 27

4.5 Potential generalizations 28

5. Appendix: physical modes 30

\section{Introduction and summary of results}

Unraveling the phase diagram of QCD, in particular at non-zero baryon density, remains a major challenge (see [1] for a recent review). Although lattice simulations have provided us with a great deal of information about the finite-temperature behavior of QCD, it is technically much less clear how to reliably extract the physics at finite baryon chemical potential, because of the notorious sign problem. One way to get around this problem is to consider non-zero isospin chemical potential instead $[2,3]$. Although there are no physical systems in nature in which only a large isospin density is achieved (because weak decays do not conserve isospin), studying such systems may nevertheless provide us with useful knowledge about the full phase diagram of QCD. ${ }^{1}$

The effects of a small isospin chemical potential $\mu_{I}$ can be analyzed using the chiral Lagrangian [3]. For chemical potentials below the mass $m_{\pi}$ of the pion, one finds that the

\footnotetext{
${ }^{1}$ Neutron stars have a large isospin density, but in this case the baryon density is also large, in contrast to the idealized situation studied here, in which the chemical potential and charge densities are turned on only in a $U(1)$ subgroup of the $S U(2)$ isospin group.
} 
pion masses (as well as the masses of other isospin triplets) split: one of them increasing, one decreasing and one remaining constant. The ground state, however, remains unmodified from that of QCD at $\mu_{I}=0$, until $\mu_{I}$ reaches the pion mass $m_{\pi}$. At this point, one of the charged pions condenses, with a magnitude that grows with $\mu_{I}$. This situation persists until $\mu_{I}$ becomes of the order of the mass of the first massive meson, where chiral perturbation theory breaks down, and it is not clear how to analyze what happens for larger values of $\mu_{I}$ (except by lattice simulations, but these are hard to perform for large pion condensates). One may consider toy models in which higher-derivative interactions or interactions with massive vectors are added, such as the Skyrme model, but it is not clear how to do this in a controllable way. Only at extremely large values of $\mu_{I}$ does one regain analytic control. Here, due to asymptotic freedom, perturbation theory applies and predicts a $\left\langle\bar{u} \gamma_{5} d\right\rangle$ condensate, much like a fermion superfluid [4]. Given that the quantum numbers of the condensate are the same as those of the pion condensate for small $\mu_{I}$, it has been conjectured [4] that no phase transition occurs as $\mu_{I}$ is increased from $m_{\pi}$ to $\infty$. However, this remains a conjecture, and it has also been suggested that the rho meson may condense when the isospin chemical potential is of the order of the rho meson mass, spontaneously breaking the rotational symmetry $[5,6,7]$ (see $[8]$ for a review). So far, lattice studies are able to reproduce the results of the chiral Lagrangian (see, for instance, [9]), and studies are currently under way to explore more of the intermediate and high isospin chemical potential regimes.

In the present paper we analyze the behavior of QCD-like theories at moderate values of the isospin chemical potential, using an alternative analytic route. Instead of analyzing QCD, we will analyze the Sakai-Sugimoto model [10, 11] (see also the review [12]) ${ }^{2}$. This model has a dimensionless parameter $\lambda_{5} / R$ (we will explain the meaning of $\lambda_{5}$ and $R$ in the next section). It is an easily-analyzed weakly-curved string theory in the limit of large $\lambda_{5} / R$, while it goes over to large $N_{c}$ QCD in the opposite limit; our analysis will be limited to the former limit, where the model contains many additional degrees of freedom beyond those of QCD. For any value of the dimensionless parameter, this model is dual to a large $N_{c}$ confining $S U\left(N_{c}\right)$ gauge theory with $N_{f}$ massless flavors, which exhibits spontaneous chiral symmetry breaking. We will analyze the case of an isospin chemical potential, as opposed to a baryon chemical potential ${ }^{3}$, for three reasons. First, we will work in the large $N_{c}$ limit, and in this limit the mesons (which are the relevant degrees of freedom for the isospin chemical potential analysis) are much more similar to the mesons of QCD than the baryons (which are relevant for the baryon chemical potential analysis); in particular they are weakly interacting and have finite masses. Note also that at large baryon chemical potential the phase diagram for large $N_{c}$ is quite different than for $N_{c}=3$ [19], but the phase diagrams seem similar for the isospin chemical potential case. Second, in the Sakai-Sugimoto model, mesons are much easier to control than baryons. The light mesons correspond simply to modes of massless fields living on the D8-branes, while baryons are

\footnotetext{
${ }^{2}$ Flavor chemical potentials in other holographic models, which do not exhibit dynamical chiral symmetry breaking, were recently studied in [13].

${ }^{3} \mathrm{~A}$ baryon chemical potential in this model was recently analyzed in $[14,15,16,17,18]$.
} 
complicated solitonic objects with a size of order the string scale [20, 21, 22, 23] (or maybe even smaller), so it is not clear how to write down the full effective action describing the baryons and their condensation. Third, as mentioned above, the phase diagram for a finite isospin chemical potential can potentially be compared with lattice simulations, because the "sign problem" which plagues simulations with finite baryon chemical potential is absent $[2,3]$.

The Sakai-Sugimoto model describes a chiral gauge theory with an $S U\left(N_{f}\right)_{L} \times S U\left(N_{f}\right)_{R}$ global symmetry which is spontaneously broken at zero temperature. At finite temperature it exhibits (at zero chemical potential) three possible phases [24, 25]. In the lowtemperature phase, the model is confined and the chiral symmetry is broken to its diagonal subgroup. In the intermediate-temperature phase, which only appears for sufficiently large constituent quark mass ${ }^{4}$, the gluons deconfine but the chiral symmetry remains broken. Finally, in the high-temperature phase, the chiral symmetry is restored. The behavior of low-spin mesons, which we will focus on in this paper, is described by small fluctuations of the flavor D8-branes, which can be treated (for $N_{f} \ll N_{c}$ ) as probes embedded in the D4-brane background. The meson spectra at zero chemical potential and non-zero temperature have been analyzed in [31].

Since the model has a vanishing quark mass, the pions are massless, and upon turning on an isospin chemical potential the charged pions immediately want to condense. In the absence of a pion mass, the pion condensate is stabilized by the non-linear interactions of the chiral Lagrangian; for any non-zero value of the isospin chemical potential, the pion goes to its maximal possible value, given that it is a Nambu-Goldstone boson which lives on a compact target space. The pion condensate leads to a small technical problem in our analysis; since the pion condensate in the Sakai-Sugimoto model corresponds to the holonomy of the D8-brane gauge field in the holographic $z$ direction, one has to deal with solutions with $A_{z} \neq 0$, while usually it is more convenient to analyze this model in the $A_{z}=0$ gauge. To avoid this complication, one can perform an $S U\left(N_{f}\right)_{L} \times S U\left(N_{f}\right)_{R}$ global symmetry transformation (which is a "large gauge transformation" in the Sakai-Sugimoto model) which eliminates the pion vacuum expectation value, and then continue working in the $A_{z}=0$ gauge. This global symmetry transformation maps a vectorial isospin chemical potential with a pion condensate into an axial isospin chemical potential with no pion condensate. Thus, instead of studying the effects of the vector-like isospin chemical potential in the presence of the pion condensate, we can equivalently study the effects of an axial isospin chemical potential in the trivial $A_{z}=0$ vacuum. Using this method, we study the effects of the chemical potential as $\mu_{I}$ is increased. In particular, we determine the behavior of the masses of the low-spin mesons as a function of $\mu_{I}$. Note that axial flavor chemical potentials may be interesting in their own right for studying the full phase

\footnotetext{
${ }^{4}$ The Sakai-Sugimoto model has another dimensionless parameter, which we will denote by $L / R$ in the next section, which determines the constituent quark mass (as measured, for instance, from the spectrum [26] or decay rates [27] of high-spin mesons). This parameter has a large effect on the physics in the limit we work in, though it is expected to decouple in the QCD limit. The bare quark mass (and, consequently, also the pion mass) is zero for any value of this parameter. Some recent attempts to add non-zero bare quark masses to this model appear in [28, 29], see also [30].
} 
diagram of QCD; as mentioned above, an axial isospin chemical potential is equivalent to a vector-like isospin chemical potential by a global symmetry transformation, but one can also study an axial $U(1)$ chemical potential, and we will describe how the Sakai-Sugimoto model reacts to such a chemical potential as well.

Our results for the Sakai-Sugimoto model at low temperatures (below the deconfinement temperature) and finite isospin chemical potential (in an $S U(2)$ subgroup of the diagonal $S U\left(N_{f}\right)$ subgroup of the flavor group) are the following. For small chemical potentials we correctly reproduce the results of the chiral Lagrangian, and the charged pion condenses to its maximal value. The isospin chemical potential $\mu_{I}$ explicitly breaks the isospin symmetry to $U(1)$; the pion condensate then breaks this $U(1)$ symmetry, but in the vacuum with the pion condensate there is a new $U(1)$ symmetry which is an axial subgroup of $U\left(N_{f}\right)_{L} \times U\left(N_{f}\right)_{R}$ which is unbroken. In this phase the isospin charge density is exactly linear in $\mu_{I}$. The interesting physics starts to appear when the chemical potential becomes of the order of the $\rho$ meson mass. In this paper we analyze this physics for the special value of the parameters $L=\pi R$, where the D8-branes are anti-podal to the anti-D8-branes. We find that there is a critical value of $\mu_{I}=\mu_{\text {crit }} \approx 1.7 m_{\rho}$ where the $\rho$ meson becomes unstable and condenses. This condensate breaks the rotational invariance from $S O(3)$ to $S O(2)$, and also breaks the remaining $U(1)$ symmetry completely. We numerically construct the new ground state, and find that for $\mu_{I}$ slightly larger than $\mu_{\text {crit }}$, the condensate behaves as $\langle\rho\rangle \propto \sqrt{\mu_{I}-\mu_{\text {crit }}}$, as expected for a second order phase transition. On top of the $\rho$ meson condensate, the pion condensate persists as well. The analysis of larger values of $\mu_{I}$, as well as of other values of $L / R$ (where there is also a non-trivial intermediate temperature phase ${ }^{5}$ ), is postponed to future work. In particular, it would be interesting to check the stability of the new state we find, and to see if additional mesons also condense as $\mu_{I}$ is further increased. In the Sakai-Sugimoto model (as in all weakly curved holographic models) the mesons of spin larger than one are much heavier (by a factor of $\sqrt{\lambda_{5} / R}$ ) than the low-spin mesons; however, when $\mu_{I}$ reaches the scale of the mass of these mesons, they may also want to condense. Our analysis is performed in the approximation of $\mu_{I} \ll \lambda_{5} / R^{2}$; for larger values of $\mu_{I}$ one has to include also DBI and Chern-Simons corrections to the D8-brane effective action, and it would be interesting to see if these wash out the rho meson condensate that we found or not.

Let us end with a few remarks concerning the relevance of our analysis for real (large $N_{c}$ ) QCD. The large isospin chemical potential analysis in QCD reviewed above, and the subsequent conjecture about the absence of phase transitions [4], crucially depends on the underlying asymptotic freedom of the theory. The Sakai-Sugimoto model, however, does not exhibit asymptotic freedom (except in its QCD limit), so apriori it is not clear how

\footnotetext{
${ }^{5}$ The analysis of isospin chemical potentials in the high-temperature phase of the Sakai-Sugimoto model is a straightforward generalization of the analysis of [32, 33]; in this phase the dynamics involves a condensation of deconfined quarks, and there is no difference between the isospin and baryon chemical potentials, as well as between vector-like and axial-like chemical potentials. Isospin chemical potentials in the intermediate and high temperature phases of the Sakai-Sugimoto model were recently analyzed in [34], but the results there do not agree with ours.
} 
this model behaves even for large $\mu_{I}$. We do not know if the phase transition described in the previous paragraph persists when we go to the QCD limit of small $\lambda_{5} / R$ or not. However, it is plausible $[5,6,7]$ that a similar phase transition could occur also in QCD in the regime of intermediate $\mu_{I}$ (of order the $\rho$ meson mass), where the analysis of [4] does not apply. It would be interesting to compute corrections to our analysis in inverse powers of $\lambda_{5} / R$ (related to $\alpha^{\prime}$ corrections), to see if these tend to strengthen or weaken the rho meson condensate, as we go in the direction of approaching QCD.

We begin in section 2 with a review of the Sakai-Sugimoto model, focussing on the realization of chiral symmetry and on the corresponding moduli space of vacua. In section 3 we analyze the isotropic solutions with chemical potentials, and compare them (for small chemical potentials) to the expectations from the chiral Lagrangian. In section 4 we analyze the stability of the isotropic solutions, and find that there is a critical value of the chemical potential where they become unstable; above that value we construct a new branch of solutions in which the rotational symmetry is spontaneously broken. An appendix contains a discussion of some technical issues in the numerical analysis.

\section{The configurations with no chemical potential}

\subsection{Review of the phases of the Sakai-Sugimoto model}

The Sakai-Sugimoto model [10] is the only known example of a holographic dual for a $3+1$ dimensional gauge theory with a continuous $S U\left(N_{f}\right)_{L} \times S U\left(N_{f}\right)_{R}$ chiral symmetry which is spontaneously broken. It is derived by considering the decoupling limit of $N_{c} \mathrm{D} 4$ branes, compactified on a circle of radius $R\left(x_{4} \equiv x_{4}+2 \pi R\right)$ with anti-periodic boundary conditions for the fermions [35], intersecting with $N_{f}$ D8-branes at $x_{4}=0$ and $N_{f}$ antiD8-branes at $x_{4}=L$. On the field theory side this gives a $4+1$ dimensional $S U\left(N_{c}\right)$ maximally supersymmetric gauge theory (with a specific UV completion which will not be relevant for us), compactified on a circle with anti-periodic boundary conditions for the adjoint fermions, and coupled to $N_{f}$ left-handed fermions in the fundamental representation of $S U\left(N_{c}\right)$ localized at $x_{4}=0$, and to $N_{f}$ right-handed fermions in the fundamental representation localized at $x_{4}=L$. In this section we will briefly review the holographic description of this model (see [10,24] for more details).

The holographic dual description of this model involves (in the large $N_{c}$ limit with fixed $\left.N_{f}\right) N_{f}$ probe D8-branes in a closed type IIA string background with a metric, Ramond-Ramond 4-form and string coupling given by

$$
\begin{aligned}
& \mathrm{d} s^{2}=\left(\frac{u}{R_{D 4}}\right)^{3 / 2}\left[-\mathrm{d} t^{2}+\delta_{i j} \mathrm{~d} x^{i} \mathrm{~d} x^{j}+f(u) \mathrm{d} x_{4}^{2}\right]+\left(\frac{R_{D 4}}{u}\right)^{3 / 2}\left[\frac{\mathrm{d} u^{2}}{f(u)}+u^{2} \mathrm{~d} \Omega_{4}^{2}\right], \\
& F_{(4)}=\frac{2 \pi N_{c}}{V_{4}} \epsilon_{4}, \quad e^{\phi}=g_{s}\left(\frac{u}{R_{D 4}}\right)^{3 / 4}, \quad R_{D 4}^{3} \equiv \pi g_{s} N_{c} l_{s}^{3}, \quad f(u) \equiv 1-\left(\frac{u_{\Lambda}}{u}\right)^{3},
\end{aligned}
$$

where $\mathrm{d} \Omega_{4}^{2}$ is the metric of a unit four-sphere and $\epsilon_{4}$ is its volume form, and $g_{s}$ is related to the $4+1$ dimensional gauge coupling by $g_{5}^{2}=(2 \pi)^{2} g_{s} l_{s}$. The submanifold spanned by $x_{4}$ and $u$ has the topology of a cigar with $u \geq u_{\Lambda}$, and requiring that this has a non-singular 
geometry gives a relation between $u_{\Lambda}$ and $R$,

$$
R=\frac{2}{3}\left(\frac{R_{D 4}^{3}}{u_{\Lambda}}\right)^{1 / 2} .
$$

The background is weakly curved and can be well-approximated by gravity whenever the 't Hooft coupling $\lambda_{5}=g_{5}^{2} N_{c}$ of the $4+1$ dimensional gauge theory is much larger than $R$. In the rest of this paper we will work in this regime. Note that in this regime the mass gap is of the same order as the scale $1 / R$ of the Kaluza-Klein modes in the gauge theory, so the $3+1$ dimensional theory does not decouple from the higher Kaluza-Klein modes (as it does in the opposite limit of $\lambda_{5} \ll R$, which is equivalent to large $N_{c}$ QCD at energies much smaller than $1 / R$ ). In this regime the confining string tension $T_{s} \sim \lambda_{5} / R^{3}$ is much larger than the scale of the mass gap, enabling an approximation in which the higher string excitations are ignored.

The probe D8-branes fill the $3+1$ dimensional space-time and the four-sphere, and trace a curve $u\left(x_{4}\right)$ in the remaining two coordinates. This curve is a solution to the equations of motion of the probe D8-branes, with the boundary condition that $u \rightarrow \infty$ at $x_{4}=0$ (with one orientation of the D8-branes) and at $x_{4}=L$ (with the opposite orientation). At $x_{4}=L / 2$ the solution reaches its minimal value of $u$, which we denote by $u=u_{0}$. In the special case of $L=\pi R, u_{0}=u_{\Lambda}$ and there is a simple solution in which $u$ is independent of $x_{4}$, with a brane at $x_{4}=0$ joining smoothly (at $u=u_{\Lambda}$ ) with an anti-brane at $x_{4}=\pi R$. In other cases the solution cannot be written down explicitly, but it satisfies the first order differential equation

$$
\frac{u^{4} f(u)}{\sqrt{f(u)+\left(\frac{R_{D 4}}{u}\right)^{3} \frac{u^{\prime 2}}{f(u)}}}=u_{0}^{4} \sqrt{f\left(u_{0}\right)} .
$$

As we will review below, the fact that the D8-branes and anti-D8-branes join together smoothly is a reflection of the spontaneous breaking of the (classical) $U\left(N_{f}\right)_{L} \times U\left(N_{f}\right)_{R}$ chiral symmetry to the diagonal $U\left(N_{f}\right)_{V}$.

At finite temperature $T$ we can describe the theory by a solution of the equations of motion in Euclidean space. There are two possibilities for the closed string background. For $T<1 / 2 \pi R$ the dominant solution is simply the Euclidean continuation of (2.1), with the time direction periodically identified $t \equiv t+1 / T$. In this solution the $x_{4}$ circle shrinks to zero size at $u=u_{\Lambda}$ while the Euclidean time circle never shrinks (see the left-hand side of figure (1). For $T>1 / 2 \pi R$ the solution is the same but with the roles of $t$ and $x_{4}$ interchanged, such that the $f(u)$ factor in the metric sits in front of $\mathrm{d} t^{2}$. In this phase $u_{\Lambda}$ is related to the periodicity in the Euclidean time direction,

$$
\frac{1}{T}=\frac{4 \pi}{3}\left(\frac{R_{D 4}^{3}}{u_{\Lambda}}\right)^{1 / 2} .
$$

In the low-temperature phase $T<1 / 2 \pi R$ the profile of the D8-branes remains exactly the same as at zero temperature. At higher temperatures there are two possible profiles 


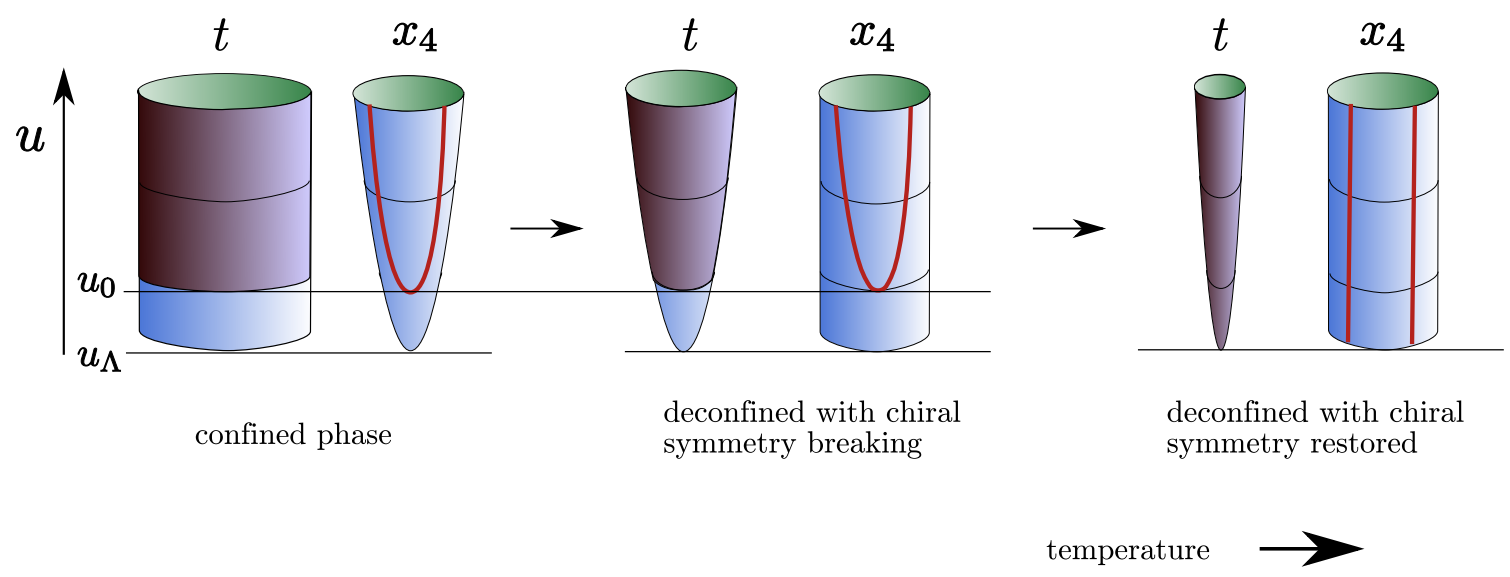

Figure 1: The topologies of the background and of the D8-branes in the three phases of the Sakai-Sugimoto model. For $L>0.97 R$, the model jumps directly from the first phase to the third phase at $T=1 / 2 \pi R$.

for the D8-branes $[24,25]$. For $1 / 2 \pi R<T<0.154 / L$ (the "intermediate temperature phase"), the dominant profile is similar to the one above, and the branes join together and spontaneously break the chiral symmetry (see the middle of figure 1). The function $u\left(x_{4}\right)$ obeys a very similar equation to the one above,

$$
\frac{u^{4} \sqrt{f(u)}}{\sqrt{1+\left(\frac{R_{D 4}}{u}\right)^{3} \frac{u^{\prime 2}}{f(u)}}}=u_{0}^{4} \sqrt{f\left(u_{0}\right)} .
$$

On the other hand, for $T>0.154 / L$ and $T>1 / 2 \pi R$ (the "high temperature phase"), the dominant configuration is that of separate branes at $x_{4}=0$ and anti-branes at $x_{4}=L$, which smoothly end at the horizon $u=u_{\Lambda}$ (see the right-hand side of figure 1). In this phase the chiral symmetry is restored.

\subsection{The chiral symmetry and the moduli space}

In holographic dualities (generalizing the AdS/CFT correspondence [36]) there is a oneto-one mapping between gauge symmetries in the bulk and global symmetries of the dual field theory. More precisely, any gauge field $A_{\mu}(x, u)$ (where $x$ are the coordinates of the dual field theory, $u$ is the radial direction and we assume that we have performed a KaluzaKlein reduction over any additional compact dimensions) that is present near the boundary $u \rightarrow \infty$ corresponds to a generator of the global symmetry of the theory. The theory is invariant under gauge transformations

$$
A_{\mu} \rightarrow g A_{\mu} g^{-1}+i g \partial_{\mu} g^{-1}
$$

with a gauge parameter $g(x, u)$ (an element of the gauge group $G$ ) which goes to the identity element $g=I$ at the boundary. "Large gauge transformations" in which $g(x, u \rightarrow \infty)=g_{0}$ 
correspond to global symmetry transformations with parameter $g_{0}{ }^{6}$. If the vacuum is invariant under such a "large gauge transformation" then the global symmetry is unbroken, and otherwise it is broken.

This correspondence applies both to gauge fields coming from closed strings and to gauge fields coming from open strings. In the D8-brane configurations we discussed above, there are two independent $U\left(N_{f}\right)$ gauge fields living near the boundary of space-time; one gauge field $\left(A_{\mu}^{L}\right)$ living at $x_{4}=0$ and another $\left(A_{\mu}^{R}\right)$ living at $x_{4}=L$. Thus, these theories have a $U\left(N_{f}\right)_{L} \times U\left(N_{f}\right)_{R}$ global symmetry ${ }^{7}$. Note that in this case the gauge fields near the boundary are only functions of $3+1$ out of the $4+1$ dimensions of the gauge theory, reflecting the fact that the global symmetry acts on fields which are localized in $3+1$ dimensions.

In a configuration where the D8-branes are separate from the anti-D8-branes, as we have in the "high temperature phase", we can perform separate constant gauge transformations (of the form (2.6) with $g=g_{0} \in U\left(N_{f}\right)$ ) for the fields $A_{\mu}^{L}$ and $A_{\mu}^{R}$, and the theory is invariant under these transformations. Thus, in this phase the full global symmetry is unbroken.

On the other hand, in a phase where the D8-branes and anti-D8-branes are connected, the fields $A_{\mu}^{L}$ and $A_{\mu}^{R}$ are limits of a single gauge field living on the D8-branes, and we cannot perform independent gauge transformations on each of them separately. We can still perform a constant gauge transformation $g=g_{0}$ on the full gauge field $A_{\mu}(\vec{x}, z)$ (here $z$ is a single-valued coordinate on the D8-branes, such that $z \rightarrow-\infty$ as the D8-branes approach the boundary at $x_{4}=0$, and $z \rightarrow \infty$ as the D8-branes approaches the boundary at $x_{4}=L$; see $\$ 3.3$ for details). Near the boundary this acts on both $A_{\mu}^{L}$ and $A_{\mu}^{R}$ by the same gauge transformation $g_{0}$, so it corresponds to the vector-like global symmetry in the theory. If we start from a configuration of the D8-branes which has a vanishing gauge field $A_{\mu}=0$, then this transformation leaves this configuration invariant, meaning that the vector-like symmetry is unbroken.

However, suppose that we want to act with a non-vector-like global symmetry transformation, in which the transformation on $A_{\mu}^{L}$ goes to $g_{L} \in U\left(N_{f}\right)$ near the boundary, while that of $A_{\mu}^{R}$ goes to $g_{R} \in U\left(N_{f}\right)$ (with $g_{R} \neq g_{L}$ ). We cannot achieve such a transformation with a gauge parameter $g(x, z)$ which is a constant. The simplest possibility is to choose $g=g(z)$, such that $g$ is a smooth function with $g(-\infty)=g_{L}$ and $g(\infty)=g_{R}$. However, such a gauge transformation does not leave our configuration invariant, since it generates $A_{z}=i g \partial_{z} g^{-1}$ which is non-vanishing. In particular, the gauge-invariant holonomy

$$
U=P \exp \left(i \int_{-\infty}^{\infty} \mathrm{d} z A_{z}\right)
$$

\footnotetext{
${ }^{6}$ If the $u \rightarrow \infty$ limit of $g(x, u)$ gives a result which depends on $x$, then the transformation is not a symmetry at all.

${ }^{7}$ The axial $U(1)$ symmetry is actually anomalous so it is not a global symmetry. However, in this paper we will only discuss the classical theory in the bulk (the leading order in $1 / N_{c}$ ) so this will not be relevant for us $[37,38,10,39]$.
} 
which was the identity matrix $U=I$ before this transformation, becomes $U=g_{L}^{-1} g_{R}$ after this transformation. Thus, the theory is not invariant under global symmetry transformations with $g_{L} \neq g_{R}$, reflecting the spontaneous breaking of the chiral symmetry.

Of course, even when the global symmetry is spontaneously broken, a global symmetry transformation still takes us from one vacuum to another, equivalent, vacuum of the theory. The theory we are discussing has a (classical) moduli space of vacua $\left(U\left(N_{f}\right) \times U\left(N_{f}\right)\right) / U\left(N_{f}\right)$, and we can go from any vacuum to any other vacuum by a global symmetry transformation. Usually one characterizes the vacua by a matrix $U$ which transforms under the global symmetry as $U \rightarrow g_{L}^{-1} U g_{R}$; we see that in the Sakai-Sugimoto model this matrix is precisely the holonomy (2.7) we discussed above ${ }^{8}$. The fluctuations in $U$ are usually written as $U=\exp \left(i \pi_{a}(x) T^{a} / f_{\pi}\right)$, where $T^{a}$ are the generators of $U\left(N_{f}\right)$ and the "pions" $\pi_{a}(x)$ are the Nambu-Goldstone bosons; in the Sakai-Sugimoto model these particles come from fluctuations of the lowest radial mode of $A_{z}$ (this statement depends on the gauge; for instance, in the $U=I$ vacuum one could choose the gauge $A_{z}=0$ and then the pions would come from the boundary conditions on the other components of $A_{\mu}$ instead $[10,11])$.

Since all the vacua of the theory are equivalent, it is usually enough to study the vacuum $U=I$ which is described by the solution $A_{\mu}=0$. However, in this paper we will be interested in turning on deformations which explicitly break the global symmetry. Such deformations destroy the equivalence between the different vacua, so one should study their effect separately in each vacuum. Nevertheless, since all the vacua are related by the global symmetry, we can always study the effect of the deformation on a vacuum with some nontrivial $U=g_{L}^{-1} g_{R}$ by performing the transformation back to the vacuum $U=I$, but taking into account the fact that the global symmetry transformation acts also on the deformation. Thus, instead of studying the effect of the deformation as a function of the vacuum, we can stay in the vacuum $U=I$ and study the effect of the deformation as a function of a global symmetry transformation $\left(g_{L}, g_{R}\right)$ acting on the deformation parameters.

\section{Isotropic solutions with flavor chemical potentials}

In this section we will add isospin chemical potentials to the model described in the previous section. We begin in $\$ 3.1$ with a general discussion of chemical potentials in the SakaiSugimoto model. We continue in $\$ 3.2$ with an analysis of the effect of chemical potentials on the chiral Lagrangian, which is the low-energy limit of our model. In $\S 3.3$ and $\S 3.4$ we find isotropic solutions of the full Sakai-Sugimoto model with chemical potentials, and we show that they reproduce our expectations from the chiral Lagrangian.

\subsection{Flavor chemical potentials in the Sakai-Sugimoto model}

As alluded to in the previous section, a gauge field $A_{\mu}$ in the bulk of a holographic model is dual to a global symmetry current $J^{\mu}$ in the field theory. As for any other field in the bulk,

\footnotetext{
${ }^{8}$ Note that in the other vacua the preserved symmetry is not the vector-like subgroup of $U\left(N_{f}\right) \times U\left(N_{f}\right)$ but a different subgroup, defined by $g_{L}^{-1} U g_{R}=U$.
} 
the behavior of the gauge field near the boundary includes independent non-normalizable and normalizable terms; in the Sakai-Sugimoto model this behavior is given by (as $u \rightarrow \infty$, in the gauge $A_{u}=0$ )

$$
A_{\nu}(x, u) \rightarrow B_{\nu}(x)\left(1+\mathcal{O}\left(\frac{1}{u}\right)\right)+\rho_{\nu}(x) u^{-3 / 2}\left(1+\mathcal{O}\left(\frac{1}{u}\right)\right) .
$$

From here on we use conventions with $\mu, \nu=0,1,2,3, i, j=1,2,3$. Again, as for any other field, the coefficient $B_{\nu}(x)$ of the non-normalizable mode is interpreted as a source term in the Lagrangian of the field theory of the form $\int \mathrm{d}^{4} x B_{\nu}(x) J^{\nu}(x)$, while $\rho_{\nu}(x)$ is proportional to the vacuum expectation value of $J_{\nu}(x)$. The freedom in performing gauge transformations in the bulk does not affect the source term in the Lagrangian due to the conservation equation $\partial_{\nu} J^{\nu}(x)=0$. Adding a chemical potential to the field theory corresponds to adding a constant source for $J^{0}$, so it corresponds to boundary conditions of the form (3.1) with $B_{\nu}(x)=\mu \delta_{\nu, 0}$.

As described above, the gauge field living on the D8-branes in the Sakai-Sugimoto model comes near the boundary at two independent positions, which we denoted $z \rightarrow-\infty$ and $z \rightarrow \infty$. Thus, for this gauge field we have two independent chemical potentials $A_{\nu}(x, z \rightarrow-\infty)=\mu_{L} \delta_{\nu, 0}$ and $A_{\nu}(x, z \rightarrow \infty)=\mu_{R} \delta_{\nu, 0}$, where $\mu_{L}$ and $\mu_{R}$ are matrices in the adjoint of $U\left(N_{f}\right)_{L}$ and $U\left(N_{f}\right)_{R}$, respectively ${ }^{9}$.

In the high-temperature phase, we have two separate stacks of D8-branes. The stack at $x_{4}=0$ only feels $\mu_{L}$ and reacts to it, and the stack at $x_{4}=L$ only feels $\mu_{R}$ and reacts to it. These chemical potentials lead to a non-trivial gauge field on the D8-branes which leads to a specific charge density $\rho_{0}(x)$ which is linear in the chemical potential (for small $\mu$ ), as described in [32, 33]. For small enough $\mu$ (compared to $\lambda_{5} T^{2}$ ) so that we can ignore the higher order terms in the DBI action, the solution in this phase has $F_{0 u, L}=-3 \mu_{L} u_{\Lambda}^{3 / 2} / 2 u^{5 / 2}$ and $F_{0 u, R}=-3 \mu_{R} u_{\Lambda}^{3 / 2} / 2 u^{5 / 2}$, leading to a change in the action

$$
\Delta S \propto-\operatorname{tr}\left(\mu_{L}^{2}+\mu_{R}^{2}\right) u_{\Lambda}^{3 / 2} .
$$

In this paper we will analyze the lower temperature phases, where the global symmetry is broken. It is then often convenient to change the notation from left and right chemical potentials to vector and axial chemical potentials, defined (in an arbitrary normalization) by $\mu_{L}=\mu_{V}-\mu_{A}$ and $\mu_{R}=\mu_{V}+\mu_{A}$. The vector-like chemical potential $\mu_{V}$ is even under $z \rightarrow-z$, while the axial-like chemical potential $\mu_{A}$ is odd. Note that the $U(1)$ components of $\mu_{V}$ and $\mu_{A}$ are the chemical potentials for the baryon number and the axial $U(1)$, respectively. The non-Abelian components correspond to isospin-like symmetries, either vector or axial. When studying the isospin we will usually limit ourselves to an $S U(2)$ subgroup of $U\left(N_{f}\right)$, and turn on chemical potentials proportional to $\sigma_{3}$ in this subgroup; the generalization to arbitrary chemical potentials (in the Cartan subalgebra of $S U\left(N_{f}\right)$ ) is straightforward.

Our definition of $\mu_{V}$ and $\mu_{A}$ is natural in the $U=I$ vacuum where the vector-like subgroup is unbroken (see footnote \&). Under the $U\left(N_{f}\right) \times U\left(N_{f}\right)$ global symmetry, the

\footnotetext{
${ }^{9}$ We will use conventions in which $A_{0}$ is the Lorentzian gauge field, such that $A_{0}$ and $\mu$ are real.
} 
chemical potentials transform as $\mu_{L} \rightarrow g_{L}^{-1} \mu_{L} g_{L}$ and $\mu_{R} \rightarrow g_{R}^{-1} \mu_{R} g_{R}$. The $U(1)$ components of the chemical potentials are invariant, but the $S U\left(N_{f}\right)$ components are not. As discussed at the end of $\delta 2.2$, we can rotate any vacuum to $U=I$ by an appropriate global symmetry transformation, but we have to remember to act with the same transformation also on the chemical potentials. In particular, studying the same chemical potential in different vacua translates into studying different chemical potentials in the $U=I$ vacuum.

\subsection{Chemical potentials in the chiral Lagrangian}

The spectrum of mesonic states in the Sakai-Sugimoto model at zero temperature [10] includes massless pions (which are the Nambu-Goldstone bosons of the chiral symmetry breaking) and other massive mesons. At low enough energies the theory can be described just by the effective action of the pions; on general grounds this is given by the chiral Lagrangian, which depends on the matrix $U$ which we described in the previous section (this was explicitly derived from the Sakai-Sugimoto model in [10] $)^{10}$. For large enough temperatures one expects additional degrees of freedom to become important. However, in the low temperature and intermediate temperature phases it is still true [24] that the only light states which are charged under the flavor symmetry ${ }^{11}$ are the pions, and there is a gap to any other charged states (in the low temperature phase the other states are just massive mesons, while in the intermediate temperature phase they include also massive deconfined quarks). Thus, in these phases that we are interested in, the low energy dynamics is still captured by the chiral Lagrangian. In particular, the response of the theory to small chemical potentials should also be captured by this Lagrangian. Thus, in this subsection we analyze the response of the chiral Lagrangian to chemical potentials. In the next subsection we will verify that the Sakai-Sugimoto model leads to the same results for small chemical potentials. Our discussion for vector-like chemical potentials is a special case of the discussion in [40] (with vanishing quark masses), but we will add also axial-like chemical potentials. As mentioned above (footnote 7), since we are in the large $N_{c}$ limit we will ignore the anomaly in the axial $U(1)$.

The chiral Lagrangian may be written as

$$
\mathcal{L}_{\text {chiral }}=\frac{f_{\pi}^{2}}{4} \operatorname{Tr}\left(D_{\nu} U D^{\nu} U^{\dagger}\right)
$$

The field $U$ is an $N_{f} \times N_{f}$ unitary matrix transforming under the global symmetry as $U \rightarrow g_{L}^{-1} U g_{R}$. The covariant derivative $D_{\nu}$ is just the normal derivative in the absence of

\footnotetext{
${ }^{10}$ At higher energies the effective theory of the pions includes also higher derivative terms. Including the leading higher correction, which comes just from the Yang-Mills action on the D8-branes, leads to the Skyrme model with a four-derivative term suppressed by the scale $1 / L$. Note that this scale is of the same order as the mass of the massive mesons, so at this scale one cannot necessarily trust an effective action which includes only the pions. The full effective action of the Sakai-Sugimoto model includes also many additional higher derivative terms which are governed by the confining string scale, so at energies of the order of the string scale we can no longer use any effective action.

${ }^{11}$ As discussed in the introduction, we will not include the baryon number symmetry, which behaves very differently from the other flavor symmetries, in our analysis.
} 
chemical potentials. However, in the presence of chemical potentials it is modified to

$$
\begin{aligned}
D_{\nu} U & =\partial_{\nu} U-i \delta_{\nu, 0}\left(\mu_{L} U-U \mu_{R}\right)=\partial_{\nu} U-i \delta_{\nu, 0}\left(\left[\mu_{V}, U\right]-\left\{\mu_{A}, U\right\}\right), \\
D_{\nu} U^{\dagger} & =\partial_{\nu} U^{\dagger}+i \delta_{\nu, 0}\left(U^{\dagger} \mu_{L}-\mu_{R} U^{\dagger}\right)=\partial_{\nu} U^{\dagger}-i \delta_{\nu, 0}\left(\left[\mu_{V}, U^{\dagger}\right]+\left\{\mu_{A}, U^{\dagger}\right\}\right) .
\end{aligned}
$$

Note that the baryon number chemical potential $\mu_{V} \propto I$ does not appear in the action at all; this is not surprising since none of the light states in the model carry a baryon number charge. Thus, the baryon number chemical potential has no effect on the classical low-energy action.

The potential energy arising from the Lagrangian (3.3) is

$$
V_{\text {chiral }}=\frac{f_{\pi}^{2}}{4} \operatorname{Tr}\left(\left(\left[\mu_{V}, U\right]-\left\{\mu_{A}, U\right\}\right)\left(\left[\mu_{V}, U^{\dagger}\right]+\left\{\mu_{A}, U^{\dagger}\right\}\right)\right) .
$$

The charge density is :

$$
\begin{aligned}
& \rho_{V}=\frac{\partial \mathcal{L}_{\text {chiral }}}{\partial \mu_{V}}=-\frac{f_{\pi}^{2}}{2}\left(U\left(\mu_{V}+\mu_{A}\right) U^{\dagger}+U^{\dagger}\left(\mu_{V}-\mu_{A}\right) U-2 \mu_{V}\right), \\
& \rho_{A}=\frac{\partial \mathcal{L}_{\text {chiral }}}{\partial \mu_{A}}=\frac{f_{\pi}^{2}}{2}\left(U\left(\mu_{A}+\mu_{V}\right) U^{\dagger}+U^{\dagger}\left(\mu_{A}-\mu_{V}\right) U+2 \mu_{A}\right) .
\end{aligned}
$$

We can now analyze the dynamics in various cases. We can start by having a chemical potential just for the axial $U(1), \mu_{A}=\mu_{a} I / \sqrt{N_{f}}$. In this case the potential is simply a constant $V_{\text {chiral }}=-f_{\pi}^{2} \mu_{a}^{2}$ so there is no potential generated on the moduli space, and the axial charge density is given by $\rho_{a} \equiv \operatorname{tr}\left(\rho_{A}\right) / \sqrt{N_{f}}=2 f_{\pi}^{2} \mu_{a}$. The axial $U(1)$ chemical potential has precisely the same, rather trivial, effect also when additional chemical potentials are turned on, so we will assume it is zero from here on.

Next, let us turn on a vector-like isospin chemical potential $\mu_{V}=\mu_{I} \sigma_{3} / 2$ [40]. Our potential then takes the form

$$
V_{\text {chiral }}=\frac{f_{\pi}^{2} \mu_{I}^{2}}{8} \operatorname{Tr}\left(\sigma_{3} U \sigma_{3} U^{\dagger}-1\right) .
$$

This is maximized by configurations where $U$ is along the identity and $\sigma_{3}$ directions, namely $U_{\max }=e^{i \alpha}\left(\cos (\beta) I+i \sin (\beta) \sigma_{3}\right)$, and minimized by configurations in the $\sigma_{1,2}$ directions, $U_{\text {min }}=e^{i \alpha}\left(\cos (\beta) \sigma_{1}+\sin (\beta) \sigma_{2}\right)$. Thus, the minimal energy configurations in this case will involve matrices of the form $U_{\min }$. This is natural since there are massless pions charged under the isospin symmetry, and they want to condense to their maximal possible value when we turn on the isospin chemical potential. The isospin charge density in the $U_{\text {min }}$ configurations is given by $\operatorname{tr}\left(\rho_{V} \sigma_{3}\right)=2 f_{\pi}^{2} \mu_{I}$, while in the $U_{\text {max }}$ configurations it vanishes. Note that in the vacua $U=U_{\min }$ the $U(1)$ symmetry corresponding to the chemical potential that we turn on is broken.

It is just as simple to analyze the case where we have both axial and vector isospin chemical potentials; assuming that both of them are in the $\sigma_{3}$ direction $\left(\mu_{V}=\mu_{I} \sigma_{3} / 2\right.$ and $\left.\mu_{A}=\mu_{A, I} \sigma_{3} / 2\right)$, we find a potential

$$
V_{\text {chiral }}=\frac{f_{\pi}^{2}}{8}\left[\left(\mu_{I}^{2}-\mu_{A, I}^{2}\right) \operatorname{Tr}\left(\sigma_{3} U \sigma_{3} U^{\dagger}\right)-\left(\mu_{I}^{2}+\mu_{A, I}^{2}\right) \operatorname{Tr}(1)\right] .
$$


Thus, if $\mu_{I}^{2}>\mu_{A, I}^{2}$ the minimum is still at the same $U_{\min }$, and the vacuum energy turns out to be independent of $\mu_{A, I}$, while if $\mu_{I}^{2}<\mu_{A, I}^{2}$ then the minimum is at one of the $U_{\max }$ configurations, and the vacuum energy is independent of $\mu_{I}$. In all of these cases, the vector-like charge density vanishes in the $U=I$ vacuum, while the axial-like charge density is $\operatorname{tr}\left(\rho_{A} \sigma_{3}\right)=2 f_{\pi}^{2} \mu_{A, I}$.

An important point is that, as we mentioned at the end of $\$ 2.2$, we can study all these vacua also by transforming them to the trivial vacuum $U=I$. Suppose we wish to study the theory with a purely vector-like isospin chemical potential, $\mu_{L}=\mu_{R}=\mu_{I} \sigma_{3} / 2$. As discussed above, in this case $U=I$ is a maximum of the scalar potential, so it corresponds to an unstable solution to the equations of motion. The stable solutions are at $U=U_{\min }$. We can transform this to $U=I$ in various ways. For example, we can choose $g_{L}=U_{\min }$, $g_{R}=I$; this transforms the vacuum to $U=I$, and the chemical potentials to a purely axial form $\mu_{L}=-\mu_{R}=-\mu_{I} \sigma_{3} / 2$. Other choices give similar results (related by the $U\left(N_{f}\right)_{V}$ symmetry which is unbroken in the $U=I$ vacuum). Thus, we can study the behavior at the minima of the scalar potential with a vector-like isospin chemical potential by analyzing the vacuum $U=I$ with a purely axial isospin chemical potential, see figure 2. Note that this is consistent with the fact that $U=I$ is a minimum of the potential in the latter case. ${ }^{12}$ Note also that even though the pion condensate breaks the original $U(1)$ symmetry preserved by a purely vector-like isospin chemical potential, there is a new $U(1)$ global symmetry which is unbroken in each of the vacua $U=U_{\min }$; after the global symmetry transformation to $U=I$ this is simply the vector-like $U(1)$ subgroup of the isospin symmetry (but in the original variables it is an axial-like symmetry).

\subsection{Isotropic solutions for small chemical potentials}

In this section we will look for solutions to the equations of motion of the Sakai-Sugimoto model in the presence of the various chemical potentials discussed above. As discussed in $\S 3.1$, such solutions involve configurations in which $A_{0}$ is non-vanishing. In this section we will only look for solutions which are translationally and rotationally invariant. Such solutions depend only on the time $t$ and on the radial coordinate $z$, and must have $A_{i}=0$.

We will start with the solutions for small $\mu$, which can be compared to those of the chiral Lagrangian, and in $\$ 3.4$ we will generalize this to larger values of $\mu$. For small $\mu$ we can approximate the action for the gauge fields on the D8-brane by the Yang-Mills action; from the form of the solutions that we will find, it is easy to see that the DBI corrections to the YM action start becoming important when $\mu \sim \lambda_{5} / L^{2}$, so our solutions in this subsection are valid for $\mu \ll \lambda_{5} / L^{2}$. Moreover, we will only work to the leading non-trivial order in $\mu$, so we will ignore the backreaction of the gauge fields on the brane embedding $u\left(x_{4}\right)$ which is of order $\mu^{2}$ (this is not present when $L=\pi R$ but is present in all other cases). Since we are interested in solutions with $A_{i}=0$, the CS term will play no role, so we will ignore it in this section.

\footnotetext{
${ }^{12}$ Note also that if we transform a general value of $U$ (with a vector-like isospin chemical potential), which is neither of the form $U_{\min }$ nor of the form $U_{\max }$, to $U=I$, we would get vector-like and axial-like chemical potentials that do not commute with each other; for such chemical potentials $U=I$ is not an extremum of the scalar potential, so it is not a static solution to the equations of motion.
} 


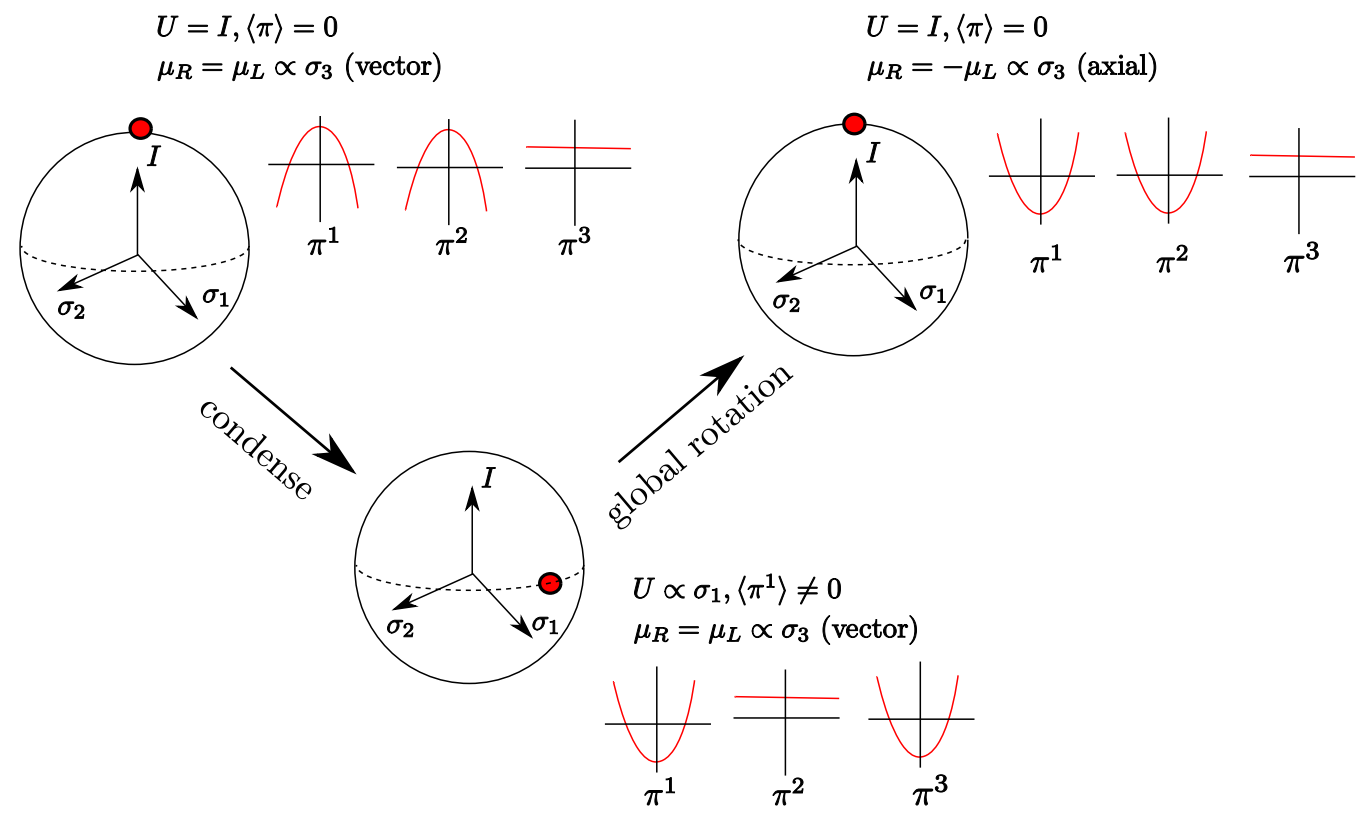

Figure 2: Introducing a vectorial isospin chemical potential leads to an instability for two of the pions (top left), and the theory wants to sit at a new minimum $U=U_{\min }$ (bottom). A global symmetry transformation brings us back to the vacuum $U=I$; this rotation changes the vector chemical potential to an axial one (top right).

As mentioned above, it is useful to choose a coordinate $z$ which is single-valued along the D8-branes; we will choose to define such a coordinate by

$$
u=\left(u_{0}^{3}+u_{0} z^{2}\right)^{1 / 3}, \quad \frac{\partial u}{\partial z}=\frac{2}{3} \frac{u_{0} z}{u^{2}},
$$

where $z$ (and $\partial u / \partial z)$ are positive for $x_{4}>L / 2$ and negative for $x_{4}<L / 2(z=0$ corresponds to the tip of the branes at $u=u_{0}$, while $z= \pm \infty$ correspond to the two asymptotic regions on the branes). Using this coordinate, we can write down the YM action density for the gauge fields on the D8-branes in the low temperature phase as

$$
S_{\mathrm{D} 8}^{\text {low }}=\tilde{T} \int_{-\infty}^{\infty} \mathrm{d} z\left[\frac{R_{D 4}^{3}|z|}{4 u^{5 / 2}} \operatorname{tr}\left(F_{\mu \nu}^{2}\right) \sqrt{\gamma}+\frac{9}{8} \frac{u^{9 / 2}}{|z| u_{0}^{2}} \operatorname{tr}\left(F_{\mu z}^{2}\right) \frac{1}{\sqrt{\gamma}}\right]
$$

with $\tilde{T}=\frac{8}{3} \hat{T}_{8} R_{D 4}^{3 / 2} u_{0}\left(2 \pi \alpha^{\prime}\right)^{2} / g_{s}$ (where, as in [24], $\hat{T}_{8}$ includes the tension of the D8-brane and the volume of the four-sphere), and

$$
\gamma(u) \equiv \frac{u^{8}}{u^{8} f(u)-u_{0}^{8} f\left(u_{0}\right)} .
$$

In the intermediate temperature phase, where the gluons are deconfined but the chiral symmetry is still broken, the action density reads

$$
S_{\mathrm{D} 8}^{\text {int }}=\tilde{T} \int_{-\infty}^{\infty} \mathrm{d} z \sqrt{f}\left[\frac{R_{D 4}^{3}|z|}{4 u^{5 / 2}}\left\{\operatorname{tr}\left(F_{i j}^{2}\right)+\frac{2}{f} \operatorname{tr}\left(F_{0 i}^{2}\right)\right\} \sqrt{\gamma}+\frac{9}{8} \frac{u^{9 / 2}}{|z| u_{0}^{2}}\left\{\frac{1}{f} \operatorname{tr}\left(F_{0 z}^{2}\right)+\operatorname{tr}\left(F_{i z}^{2}\right)\right\} \frac{1}{\sqrt{\gamma}}\right] .
$$


Note that in the limit $u_{0}=u_{\Lambda}$ these expressions simplify because then $\gamma=f^{-1}$ and $f=u_{0} z^{2} / u^{3}$. These actions assume that the brane configuration is the same as it was in the absence of the chemical potential; as mentioned above, we expect the chemical potential to generate gauge fields of order $\mu$, which should back-react on the configuration of the brane at order $\mu^{2}$.

As discussed above, we are looking for solutions with $A_{i}=0$, so the relevant part of the action is given by the $F_{0 z}^{2}$ terms, which appear with a measure $g_{2}(z)$ given by

$$
g_{2}(z)=\frac{9}{8} \frac{1}{u_{0}^{2}} \tilde{T} \times \begin{cases}\left(u_{0}^{3}+u_{0} z^{2}\right)^{3 / 2} \frac{1}{|z| \sqrt{\gamma}} & \text { low } T \\ \left(u_{0}^{3}+u_{0} z^{2}\right)^{3 / 2} \frac{1}{|z| \sqrt{f \gamma}} & \text { intermediate } T .\end{cases}
$$

The large $|z|$ behavior is obviously the same for both cases.

For simplicity, we will work in the $A_{z}=0$ gauge. As discussed above, this means that we are at a specific point $U=I$ in the moduli space, but this point is related to all other points by the global symmetry transformation so we can still discuss the most general case. Naively, going to $A_{z}=0$ gauge means that we are setting the full pion field to zero, and not just its zero modes, but in fact this is not true; as discussed in [10], in this gauge the derivatives of the pions show up through the boundary values of $A_{\mu}$.

Thus, we wish to solve the equations of motion with an ansatz of the form

$$
\begin{aligned}
& A_{z}=0, \quad A_{i}=0, \\
& A_{0}=A_{0}^{V}(z, t)+A_{0}^{A}(z, t), \quad F_{0 z}=-\partial_{z} A_{0},
\end{aligned}
$$

where we separated the field $A_{0}$ into its even part (under $z \rightarrow-z$ ) $A_{0}^{V}$ (related to the vector-like symmetry) and its odd part $A_{0}^{A}$ (related to the axial symmetry). As discussed in $\S 3.1$, for a solution with a given chemical potential, $A_{0}$ should approach $\mu_{L}$ as $z \rightarrow-\infty$ and $\mu_{R}$ as $z \rightarrow \infty$, and the coefficient of the normalizable mode of $A_{0}$ at infinity (which we called $\rho_{0}(x)$ in (3.1)), which is proportional to $1 / z$, is proportional to the charge density.

With the ansatz (3.14), the equations of motion become

$$
\partial_{0} \partial_{z} A_{0}-i\left[A_{0}, \partial_{z} A_{0}\right]=0, \quad \partial_{z}\left(g_{2}(z) \partial_{z} A_{0}\right)=0 \text {. }
$$

The second equation implies that the general solution for the field strength takes the form

$$
F_{0 z}=\frac{\tilde{f}_{A}(t)}{g_{2}(z)} \Rightarrow A_{0}=f_{A}(t) \int_{0}^{z} \frac{\mathrm{d} \tilde{z}}{g_{2}(\tilde{z})}\left(\int_{0}^{\infty} \frac{\mathrm{d} \tilde{z}^{\prime}}{g_{2}\left(\tilde{z}^{\prime}\right)}\right)^{-1}+f_{V}(t)
$$

for some $U\left(N_{f}\right)$-valued functions $f_{V}(t)$ and $f_{A}(t)$, which must obey the additional equation of motion

$$
\partial_{0} f_{A}(t)-i\left[f_{V}(t), f_{A}(t)\right]=0 .
$$

Note that the contribution of $f_{V}$ to $A_{0}$ is even under $z \rightarrow-z$ (in fact it is independent of $z$ ) so it includes any vector-like chemical potential and charge density, while the contribution of $f_{A}$ is odd and includes the axial chemical potential and charge densities. In the special case of the low temperature phase with $L=\pi R$ such that $u_{0}=u_{\Lambda}$, it is easy 
to perform the integral in (3.16) explicitly, and to write the general solution in the form $A_{0}=2 f_{A}(t) \arctan \left(z / u_{\Lambda}\right) / \pi+f_{V}(t)$ (where one still has to impose (3.17)).

Near the boundary, the solution (3.16) approaches $f_{V}(t) \pm f_{A}(t)$ at $z= \pm \infty$. In the case that the solution is time-independent, we thus identify $f_{V}=\mu_{V}$ and $f_{A}=\mu_{A}$, and we find such a solution whenever $\left[\mu_{V}, \mu_{A}\right]=0$. This agrees with the expectation described in $\S 3.2$, that for commuting $\mu_{V}$ and $\mu_{A}, U=I$ should be a static (not necessarily stable) solution. By examining the terms going as $1 / z$ near the boundary, we see that these solutions have $\rho_{V}=0$ and $\rho_{A}=C \mu_{A}$ for some constant $C$ that we will compute below. Again, this precisely agrees with the expectations described in $\$ 3.2$. Note that in the purely vector-like case the field strength vanishes identically.

The conclusions of the previous paragraph hold both for the $U(1)$ and for the $S U\left(N_{f}\right)$ components; as in the chiral model, the $U(1)$ components of the gauge field are independent of the $S U\left(N_{f}\right)$ components so we can discuss them separately. In the $S U\left(N_{f}\right)$ case, if we take $\mu_{V}=\mu_{I} \sigma_{3} / 2$ and $\mu_{A}=\mu_{A, I} \sigma_{3} / 2$, then, as discussed in $\S 3.2$, the solution we find is either a maximum or a minimum, depending on the sign of $\mu_{I}^{2}-\mu_{A, I}^{2}$. However, this does not affect the form of the solution, but just the mass spectrum of small fluctuations around the solution.

As we also discussed in $\oint 3.2$, when $\left[\mu_{V}, \mu_{A}\right] \neq 0$ we do not expect to find a static solution, and indeed we see from (3.17) that there is no such solution, but that the time derivative of $f_{A}$ must be non-zero (even as $z \rightarrow \pm \infty$ ). This implies that the time-derivative of the pion field must be non-zero in this case, consistent with the fact that we are not sitting at an extremum of the pion potential. It is easy to write simple solutions to (3.17) which describe oscillations around the minima of the pion potential, but we expect that when we include interactions (at order $1 / N_{c}$ ) such solutions will eventually decay to the minima, so they are not very interesting and we will not discuss them further.

In order to compute the precise coefficient $C$ appearing in our expression above for the charge density, we need to compute the derivative of the action of our configurations with respect to $\mu_{A}$ (it is obvious that the action is independent of $\mu_{V}$ ). By plugging (3.16) into the action density, we obtain

$$
S=2 \operatorname{Tr}\left(\mu_{A}^{2}\right)\left(\int_{0}^{\infty} \frac{\mathrm{d} \tilde{z}}{g_{2}(\tilde{z})}\right)^{-1},
$$

so that the axial charge density is given by

$$
\rho_{A}=4 \mu_{A}\left(\int_{0}^{\infty} \frac{\mathrm{d} \tilde{z}}{g_{2}(\tilde{z})}\right)^{-1} .
$$

Comparing this with our expressions of the previous section, we obtain

$$
f_{\pi}^{2}=2\left(\int_{0}^{\infty} \frac{\mathrm{d} \tilde{z}}{g_{2}(\tilde{z})}\right)^{-1} .
$$

This is precisely the same expression that one obtains by computing the kinetic term for the holonomy matrix $U$ (defined in (2.7)) in the Sakai-Sugimoto model [10]; note that a 
configuration with varying holonomy $U$ is described in this model by

$$
F_{\mu z}=-\frac{i}{g_{2}(z)} U^{-1} \partial_{\mu} U\left(\int_{-\infty}^{\infty} \frac{\mathrm{d} \tilde{z}}{g_{2}(\tilde{z})}\right)^{-1} .
$$

For $L=\pi R$ we have [10] $f_{\pi}^{2} \propto \lambda_{5} N_{c} / R^{3}$. For $L \ll R$, on the other hand, the scale $R$ drops out of the analysis and we have $f_{\pi}^{2} \propto \lambda_{5} N_{c} / L^{3}$. Since it is not clear how to directly compute the chiral condensate in the Sakai-Sugimoto model, $f_{\pi}$ is the best estimate that we have for the scale of chiral symmetry breaking in this model.

We can now use the results above to analyze the response of the theory to various chemical potentials. In the case of a baryon number chemical potential, we see that its only effect is a shift in the constant mode of $A_{0}$, which has no effect on the physics. This is as we expect (for small $\mu$ ), since we did not include in our analysis any fields which are charged under the baryon number symmetry. For vector-like isospin chemical potentials we saw that we have similar solutions with constant values of $A_{0}$; these solutions have no field strength, but they affect the theory through the commutator terms in the action. As discussed above, these solutions (with $U=I$ ) should be unstable maxima of the pion potential, and the spectrum of fluctuations around these solutions contains tachyonic modes. If we want to analyze the minima $U_{\min }$ of the pion potential with a vector-like isospin chemical potential then, as discussed above, we can transform them into the $U=I$ configuration with an axial-like chemical potential. These configurations describe a charged pion which condensed to its maximal value (it is stabilized by the non-linear interactions in the chiral Lagrangian, associated with the finite moduli space of the pions). In these configurations there is a non-trivial field strength, leading both to a non-trivial free energy density and to a non-trivial charge density, coming from the condensation of the charged pions. These configurations are expected to be stable, at least for small chemical potentials where the truncation to the chiral Lagrangian is a good approximation; we will analyze their stability at chemical potentials of the order of the mass gap in section 4 .

\subsection{Isotropic solutions for large flavor chemical potentials}

The solution (3.16) for axial chemical potentials found in the previous subsection is valid only for small $\mu$, or more precisely, for $\mu \ll \lambda_{5} / L^{2}$. Our analysis ignored both the DBI corrections to the Yang-Mills action, and the fact that the back-reaction of the gauge fields which are generated will change the brane embedding. In this subsection we will generalize this analysis to larger values of $\mu$. In general, the effective action on the D8-brane includes also additional corrections beyond the DBI action, involving derivatives of the gauge fields. In the configurations we will be discussing in this paper, the corrections coming from derivatives in the radial direction are small as long as the gravity approximation is valid, namely for $\lambda_{5} \gg R$. Corrections coming from derivatives in space-time directions will be small as long as these derivatives are smaller than the string scale $\sim \lambda_{5} / R^{3}$; at higher energies or momenta than this we must use the full string theory and we cannot use any effective action. However, for the static solutions described in this section, the DBI action is valid for arbitrarily large values of $\mu$. 
We will solve the equations of motion here for a single D8-brane; as long as the chemical potential is a diagonal matrix, the full solutions (in the $U=I$ vacuum) will be diagonal, so they will just be $N_{f}$ copies of the single brane solution.

In the low temperature phase, the relevant terms in the action for a single D8-brane are

$$
S=\frac{\hat{T}_{8}}{g_{s}} \int_{0}^{L} \mathrm{~d} x_{4} u^{4} \sqrt{f(u)} \sqrt{1+\left(\frac{R_{\mathrm{D} 4}}{u}\right)^{3} \frac{u^{\prime 2}-f(u)\left(F_{04}\right)^{2}}{f(u)^{2}}} .
$$

For simplicity we will work here in the $u$ coordinate instead of in the $z$ coordinate that we used before; the translation between the two coordinate systems is straightforward. One equation of motion can be obtained from the "Hamiltonian" associated to translations in $x_{4}$,

$$
H=\frac{u^{4} \sqrt{f(u)}}{\sqrt{1+\left(\frac{R_{\mathrm{D} 4}}{u}\right)^{3} \frac{u^{\prime 2}-f(u)\left(F_{04}\right)^{2}}{f(u)^{2}}}},
$$

which is conserved, $\partial_{4} H=0$. In addition, the equation of motion for the gauge potential $A_{0}$ implies

$$
E \equiv \frac{u^{4} F_{04}}{\left(\frac{u}{R_{\mathrm{D} 4}}\right)^{3} \sqrt{f(u)} \sqrt{1+\left(\frac{R_{\mathrm{D} 4}}{u}\right)^{3} \frac{u^{\prime 2}-f(u) F_{04}^{2}}{f(u)^{2}}}}=\text { const } .
$$

Dividing (3.24) by (3.23) we get for the electric field

$$
F_{04}=\frac{E}{H}\left(\frac{u}{R_{\mathrm{D} 4}}\right)^{3} f(u) .
$$

Note that $F_{04}$ is symmetric under $z \rightarrow-z$, which means that $F_{0 z}$ is anti-symmetric, so this is an axial-like solution ${ }^{13}$. Of course, the vector-like solution is still given simply by $A_{0}=$ const, $E=0$.

In order to express this in terms of our field theory parameters we need to find the shape $u\left(x_{4}\right)$. The brane minimum is by definition at $u=u_{0}$, where $u^{\prime}=0$. This fixes $H$ in terms of $u_{0}$ to be

$$
H=\sqrt{u_{0}^{8} f\left(u_{0}\right)+E^{2} f\left(u_{0}\right)\left(\frac{u_{0}}{R_{\mathrm{D} 4}}\right)^{3}} .
$$

We can then solve for $u^{\prime}$ from the expression for $H$, which yields

$$
u^{\prime}=\sqrt{u^{8} f(u)-u_{0}^{8} f\left(u_{0}\right)+\frac{E^{2}}{R_{\mathrm{D} 4}^{3}}\left(f(u) u^{3}-f\left(u_{0}\right) u_{0}^{3}\right)}\left(\frac{u}{R_{\mathrm{D} 4}}\right)^{3 / 2} f(u) \frac{1}{H} .
$$

\footnotetext{
${ }^{13}$ This axial solution was also obtained in [32], but incorrectly interpreted as a vector-like baryon chemical potential. When the configuration is made symmetric under $z \rightarrow-z$, the resulting gauge field is no longer continuous at the tip of the brane [33].
} 
As before, for a vector-like chemical potential the solution is simply $A_{0}=\mu_{V}$ and $F_{04}=0$. For an axial-like chemical potential ${ }^{14}$ we have the following relations between the parameters of the field theory, $\mu_{A}$ and $L$, and the parameters $E$ and $u_{0}$ of the solutions found above :

$$
\begin{aligned}
\mu_{A} & =\frac{1}{2} \int_{0}^{L} d x_{4} \partial_{4} A_{0}= \\
& =-E \int_{u_{0}}^{\infty} d u\left(\frac{u}{R_{D 4}}\right)^{3 / 2}\left[u^{8} f(u)-u_{0}^{8} f\left(u_{0}\right)+\frac{E^{2}}{R_{\mathrm{D} 4}^{3}}\left(f(u) u^{3}-f\left(u_{0}\right) u_{0}^{3}\right)\right]^{-1 / 2}, \\
L & =\int d x_{4}=2 \int_{u_{0}}^{\infty} \frac{d u}{u^{\prime}}= \\
& =2 H \int_{u_{0}}^{\infty} \frac{d u}{f(u)}\left(\frac{R_{D 4}}{u}\right)^{3 / 2}\left[u^{8} f(u)-u_{0}^{8} f\left(u_{0}\right)+\frac{E^{2}}{R_{\mathrm{D} 4}^{3}}\left(f(u) u^{3}-f\left(u_{0}\right) u_{0}^{3}\right)\right]^{-1 / 2},
\end{aligned}
$$

where in the last equation $H$ should be expressed in terms of $u_{0}$ using (3.26). It is easy to see that by expanding these solutions to leading order in $\mu$ (and in $E$ ) one reobtains the solutions we discussed in the previous subsection (3.16).

Note that if we have generic isospin chemical potentials with $N_{f}>2$ and the values of $\mu_{A}^{2}$ are not equal along the diagonal, then generally the D8-branes will no longer be overlapping but will have different functions $u\left(x_{4}\right)$ except near the boundary. This was not visible in the previous subsection since the separation starts at order $\mu^{2}$ for small $\mu$. The only case where this separation does not occur is for $L=\pi R$ (i.e. for $u_{0}=u_{\Lambda}$ ), since then constant values of $x_{4}$ always solve the equations of motion.

The analysis in the intermediate temperature phase is similar. The action is now

$$
S=\frac{\hat{T}_{8}}{g_{s}} \int \mathrm{d} x_{4} u^{4} \sqrt{f+\left(\frac{R_{\mathrm{D} 4}}{u}\right)^{3}\left(u^{\prime 2}-F_{04}^{2}\right)} .
$$

The Hamiltonian takes the form

$$
H=\frac{u^{4} f(u)}{\sqrt{f+\left(\frac{R_{\mathrm{D} 4}}{u}\right)^{3}\left(u^{\prime 2}-F_{04}^{2}\right)}},
$$

and the conserved $E$ takes the form

$$
E \equiv \frac{u^{4} F_{04}}{\left(\frac{u}{R_{\mathrm{D} 4}}\right)^{3} \sqrt{f(u)+\left(\frac{R_{\mathrm{D} 4}}{u}\right)^{3}\left(u^{\prime 2}-F_{04}^{2}\right)}}=\text { const } .
$$

Dividing (3.32) by (3.31) we get for the electric field the same expression (3.25) as before, and the expression for $H\left(u_{0}\right)$ is also identical to the previous expression (3.26). Solving for

\footnotetext{
${ }^{14}$ We remind the reader that this case is equivalent to a vector-like isospin chemical potential when the theory is expanded around the minimum of the pion potential.
} 
$u^{\prime}$ we now find

$$
u^{\prime}=\sqrt{u^{8} f(u)-u_{0}^{8} f\left(u_{0}\right)+\frac{E^{2}}{R_{\mathrm{D} 4}^{3}}\left(f(u) u^{3}-f\left(u_{0}\right) u_{0}^{3}\right)}\left(\frac{u}{R_{\mathrm{D} 4}}\right)^{3 / 2} \sqrt{f(u)} \frac{1}{H} .
$$

The relations between the parameters now take the form :

$$
\begin{gathered}
\mu_{A}=-E \int_{u_{0}}^{\infty} \mathrm{d} u \sqrt{f(u)}\left(\frac{u}{R_{D 4}}\right)^{3 / 2}\left[u^{8} f(u)-u_{0}^{8} f\left(u_{0}\right)+\frac{E^{2}}{R_{\mathrm{D} 4}^{3}}\left(f(u) u^{3}-f\left(u_{0}\right) u_{0}^{3}\right)\right]^{-1 / 2}, \\
L=2 H \int_{u_{0}}^{\infty} \frac{\mathrm{d} u}{\sqrt{f(u)}}\left(\frac{R_{D 4}}{u}\right)^{3 / 2}\left[u^{8} f(u)-u_{0}^{8} f\left(u_{0}\right)+\frac{E^{2}}{R_{\mathrm{D} 4}^{3}}\left(f(u) u^{3}-f\left(u_{0}\right) u_{0}^{3}\right)\right]^{-1 / 2} .
\end{gathered}
$$

\section{Instability of isotropic solutions and rho meson condensation}

\subsection{The fluctuation equations}

In the previous section we found the static isotropic solutions of the Sakai-Sugimoto model in the presence of flavor chemical potentials. These solutions are the unique isotropic solutions in the absence of sources, but there may exist additional non-isotropic solutions, which could dominate the thermodynamics for some values of the chemical potential. We will focus on the isospin chemical potential case. Since the model contains massive particles charged under the isospin symmetry, one might expect that for a large enough chemical potential these particles will want to condense and modify the solution, and we will see that this is indeed the case.

In order to analyze the stability in general we need to use the full DBI action, and include also the Chern-Simons term in the action of the five dimensional gauge field (which includes quadratic terms in the fluctuations around the solutions of the previous section). However, as discussed above, for $\mu \ll \lambda_{5} / L^{2}$ the DBI action can be approximated just by the Yang-Mills action, and in the same regime one can also show that the contribution of the Chern-Simons term is much smaller than that of the Yang-Mills terms, so it can also be ignored. Thus, in this section we will use the Yang-Mills action to look for instabilities, and we will see that the isotropic phase discussed in the previous section becomes unstable already for $\mu$ which obeys $\mu \ll \lambda_{5} / L^{2}$, so that the analysis is self-consistent. For simplicity, we will consider in this section only the low-temperature phase. We will also consider only the special case of $L=\pi R$ for which the equations (and the isotropic solution) simplify. It should be straightforward to generalize our analysis, but we postpone this to future work.

To analyze the stability we need to consider the spectrum of fluctuations of all the fields in the theory around our solutions. In the limit of weak curvature, we can limit our analysis to the massless fields living on the D8-branes, since the other mesons are much heavier. Because the chemical potential breaks Lorentz symmetry, there are four types of fields which have to be discussed: there are KK towers of scalars, transverse vectors, and longitudinal vectors, and in addition there are the pions. All of these except the scalars arise from the five-dimensional vector field. In order to keep the vector equations 
of motion reasonably compact, we will temporarily revert back to the $u$ coordinate system. The action (in the approximation described above) then reads

$$
S=\tilde{T} \frac{3 R_{D 4}^{3}}{8 u_{0}} \int \mathrm{d}^{4} x \mathrm{~d} u\left[u^{-1 / 2} \gamma^{1 / 2} \operatorname{Tr}\left(F_{\mu \nu} F_{\rho \sigma}\right) \eta^{\mu \rho} \eta^{\nu \sigma}+2 R_{D 4}^{-3} u^{5 / 2} \gamma^{-1 / 2} \operatorname{Tr}\left(F_{\mu u} F_{\nu u}\right) \eta^{\mu \nu}\right] .
$$

As in the previous section, we will analyze the effect of a vectorial isospin density in the $U=U_{\min }$ vacuum where the pions have condensed by performing a global symmetry transformation taking this back to the $U=I$ vacuum, in which the isospin chemical potential becomes an axial isospin chemical potential. So, we will analyze the stability of the solution constructed in $\$ 3.3$ (which we write here just for the D8-brane branch $z>0$ ),

$$
\bar{A}_{0}=\mu_{I} \frac{\sigma_{3}}{2} \bar{A}(u), \quad \bar{A}(u) \equiv \mathcal{N} \int_{u_{0}}^{u} \frac{\mathrm{d} \tilde{u}}{\tilde{u}^{5 / 2} \gamma(\tilde{u})^{-1 / 2}} \quad \Rightarrow \quad \bar{F}_{0 u}=-\mu_{I} \frac{\sigma_{3}}{2} \frac{\mathcal{N}}{u^{5 / 2} \gamma^{-1 / 2}}
$$

with $\mathcal{N}$ chosen to make $\lim _{u \rightarrow \infty} \bar{A}(u)=1$. We will continue to use the gauge $A_{u}=0$. In this gauge the expansion of the gauge field in terms of fluctuations is

$$
A_{0}=\bar{A}_{0}(u)+\delta A_{0}^{(a)}(\omega, \vec{k}, u) \sigma_{a} e^{i \omega t+i \vec{k} \cdot \vec{x}}, \quad A_{i}=\delta A_{i}^{(a)}(\omega, \vec{k}, u) \sigma_{a} e^{i \omega t+i \vec{k} \cdot \vec{x}}, \quad A_{u}=0
$$

The transverse fields coming from $\delta A_{i}(\omega, \vec{k}, u)$ will be degenerate since there is still rotational invariance, but they need no longer be degenerate with $\delta A_{0}$.

The equations of motion for the diagonal gauge field fluctuations $\delta A_{i}^{(0,3)}$ and $\delta A_{0}^{(0,3)}$ do not change upon inclusion of the chemical potential and the background (4.2); their spectra remain unmodified (in the approximation of $\mu_{I} \ll \lambda_{5} / L^{2}$ where we ignore the Chern-Simons term in the action). For the other matrix elements, the action (4.1) leads to the following equations for the spatial components,

$$
\begin{gathered}
R_{D 4}^{-3} u^{1 / 2} \gamma^{-1 / 2} \partial_{u}\left(u^{5 / 2} \gamma^{-1 / 2} \partial_{u} \delta A_{i}^{(1)}\right)+\partial^{2} \delta A_{i}^{(1)}-\partial_{i}\left(\partial_{\mu} \delta A^{\mu(1)}\right) \\
+2 \mu_{I} \bar{A}(u) \partial_{0} \delta A_{i}^{(2)}-\mu_{I} \bar{A}(u) \partial_{i} \delta A_{0}^{(2)}=-\mu_{I}^{2} \bar{A}(u)^{2} \delta A_{i}^{(1)} \\
R_{D 4}^{-3} u^{1 / 2} \gamma^{-1 / 2} \partial_{u}\left(u^{5 / 2} \gamma^{-1 / 2} \partial_{u} \delta A_{i}^{(2)}\right)+\partial^{2} \delta A_{i}^{(2)}-\partial_{i}\left(\partial_{\mu} \delta A^{\mu(2)}\right) \\
-2 \mu_{I} \bar{A}(u) \partial_{0} \delta A_{i}^{(1)}+\mu_{I} \bar{A}(u) \partial_{i} \delta A_{0}^{(1)}=-\mu_{I}^{2} \bar{A}(u)^{2} \delta A_{i}^{(2)}
\end{gathered}
$$

Here $\partial^{2} \equiv \partial_{\mu} \partial^{\mu} \rightarrow \omega^{2}-\vec{k}^{2}$ and $F_{\mu \nu}=\partial_{\mu} A_{\nu}-\partial_{\nu} A_{\mu}-i\left[A_{\mu}, A_{\nu}\right]$, so that with our convention $A_{\mu}=A_{\mu}^{(a)} \sigma_{a}$ we have $F_{\mu \nu}^{(a)}=\partial_{\mu} A_{\nu}^{(a)}-\partial_{\nu} A_{\mu}^{(a)}+2 \epsilon^{a b c} A_{\mu}^{(b)} A_{\nu}^{(c)}$. In addition, we have the 
equations for the time-like and $u$-components of the gauge field, of the form

$$
\begin{gathered}
R_{D 4}^{-3} u^{1 / 2} \gamma^{-1 / 2} \partial_{u}\left(u^{5 / 2} \gamma^{-1 / 2} \partial_{u} \delta A_{0}^{(1)}\right) \\
+\left(\partial_{i} \partial^{i} \delta A_{0}^{(1)}-\partial_{0}\left(\partial_{i} \delta A_{i}^{(1)}\right)+\mu_{I} \partial_{i} \delta A_{i}^{(2)} \bar{A}(u)\right)=0, \\
R_{D 4}^{-3} u^{1 / 2} \gamma^{-1 / 2} \partial_{u}\left(u^{5 / 2} \gamma^{-1 / 2} \partial_{u} \delta A_{0}^{(2)}\right) \\
+\left(\partial_{i} \partial^{i} \delta A_{0}^{(2)}-\partial_{0}\left(\partial_{i} \delta A_{i}^{(2)}\right)-\mu_{I} \partial_{i} \delta A_{i}^{(1)} \bar{A}(u)\right)=0, \\
\partial_{u}\left(\partial_{0} \delta A_{0}^{(1)}-\partial_{i} \delta A_{i}^{(1)}\right)-\mu_{I} \bar{A}(u) \partial_{u} \delta A_{0}^{(2)}+\mu_{I} \delta A_{0}^{(2)} \bar{A}^{\prime}(u)=0, \\
\partial_{u}\left(\partial_{0} \delta A_{0}^{(2)}-\partial_{i} \delta A_{i}^{(2)}\right)+\mu_{I} \bar{A}(u) \partial_{u} \delta A_{0}^{(1)}-\mu_{I} \delta A_{0}^{(1)} \bar{A}^{\prime}(u)=0 .
\end{gathered}
$$

The equations of motion for the scalar fields will be discussed in the next subsection.

Naively, one may expect that for positive $\mu_{I}$ the masses of the fields carrying positive isospin charge would go down and the masses of the fields carrying negative isospin charge would go up (at least for small $\mu_{I}$ ). The equations above are indeed symmetric under $\mu_{I} \rightarrow-\mu_{I}, A^{(2)} \rightarrow-A^{(2)}$ which changes the sign of the isospin chemical potential and also exchanges the positively and negatively charged mesons. However, there is an extra symmetry of the equations (and of the solution (4.2)) under $\mu_{I} \rightarrow-\mu_{I}$ together with $z \rightarrow-z$ (when the equations are written in the $z$ coordinate), which implies that in fact the spectrum is the same for positive and for negative $\mu_{I}$ (note that together with the previous symmetry, this implies that the spectrum is the same for the positively charged mesons as for the negatively charged mesons). The reason for this is the condensation of the pions; the expectation above holds when we expand around the trivial vacuum $U=I$, but it does not hold when we expand around the vacuum in which the pions have condensed (or, equivalently, when we expand around the vacuum $U=I$ with an axial isospin chemical potential). Note in particular that the mesons that we analyze in this section carry a $U(1)$ charge in the new vacuum, but this is not the same as the original $U(1)$ isospin charge to which the chemical potential was coupled ${ }^{15}$. In any case, we will see that the masses of some of the fields (of both positive and negative $U(1)$ charge) go down with $\mu_{I}$, and that eventually they condense. Because of the symmetries discussed above, it is enough to consider positive values of $\mu_{I}$, and to consider either the positively charged or the negatively charged mesons (but not both), and we will use this when solving the equations.

\subsection{Transverse vectors and scalars}

The analysis of the transverse vectors and the scalars is similar to their analysis without the chemical potential [10]. Let us first focus on the solutions of the $\delta A_{i}^{(1,2)}$ fluctuations, which describe massive transverse vector mesons. For these modes, it is consistent to set $\delta A_{0}=0$ and then also impose the standard constraint for massive transverse vectors $\partial_{i} \delta A^{i}=0$. This automatically takes care of equations 4.6)-(4.9). The spectrum of eigenfunctions $A_{i}^{(1,2)}$

\footnotetext{
${ }^{15}$ In the original variables, if we choose $U_{\min } \propto \sigma_{1}$, the equations above involve the $\sigma_{1}$ and $\sigma_{3}$ components of the matrices.
} 

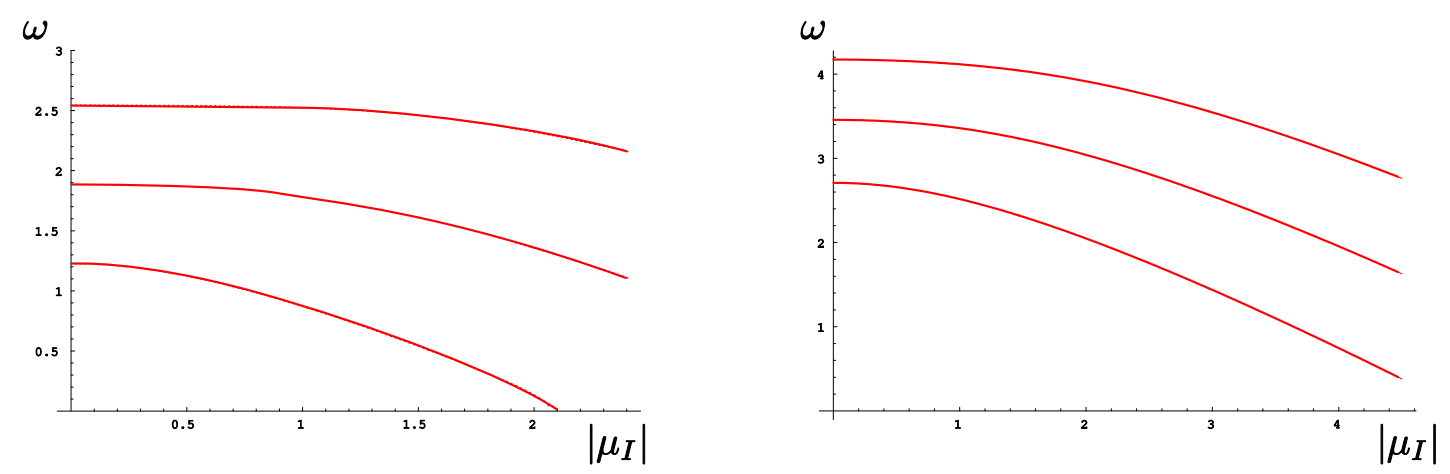

Figure 3: The frequencies of zero spatial momentum solutions for the transverse vectors (left) and scalars (right), as a function of the isospin chemical potential, in units of $\sqrt{u_{\Lambda} / R_{\mathrm{D} 4}^{3}}=2 /(3 R)$. The frequencies at $\mu_{I}=0$ reproduce the spectrum found in [10].

of (4.4) and (4.5) and the corresponding eigenvalues $\omega$ can be found numerically, using standard shooting methods, as a function of $\mu_{I}$. For the special case of $L=\pi R$ they are displayed on the left-hand side of figure 3 , in units of $\sqrt{u_{\Lambda} / R_{D 4}^{3}}=2 /(3 R)$. We see that the frequency of the lowest transverse vector goes to zero at a chemical potential of magnitude $\mu_{\text {crit }} \approx 2.1 * 2 /(3 R) \approx 1.7 m_{\rho}$, indicating a potential instability there.

A similar story to the one above holds for the scalar mesons, arising from the scalar field $y$ on the D8-brane ${ }^{16}$. The scalar mesons feel the chemical potential through the commutators in the covariant derivatives. The equation of motion for the scalars in the $I$ and $\sigma_{3}$ directions are unaffected by the chemical potential (4.2), while the equations for the $\sigma_{1}$ and $\sigma_{2}$ components are easily generalized from those in [10]. In the $z$-coordinate system they read (for $L=\pi R$ )

$$
\frac{1}{R^{2}} K^{1 / 3}\left[u_{\Lambda}^{2} \partial_{z}\left(K \partial_{z} y^{(1,2)}\right)-2 y^{(1,2)}\right]+\left(\omega^{2}-k^{2}\right) y^{(1,2)}= \pm 2 i \mu_{I} \omega \bar{A} y^{(2,1)}-\mu_{I}^{2} \bar{A}^{2} y^{(1,2)}
$$

where $K(z) \equiv\left(u / u_{\Lambda}\right)^{3}$, and where the plus/minus sign refers to the $y^{(1)}$ and $y^{(2)}$ functions, respectively. These equations are diagonal in the basis of positively and negatively charged mesons, given by

$$
y^{(1)}= \pm i y^{(2)} .
$$

The resulting behavior of the $\omega$ eigenvalues as a function of chemical potential is displayed on the right-hand side of figure 3. We observe that the scalars also go to zero frequency leading to potential instabilities, but only for chemical potentials which are larger in magnitude than those for which the first vector meson becomes unstable, so the vector instability must be analyzed first.

\footnotetext{
${ }^{16}$ There are additional scalar fields coming from the components of the gauge field along the $S^{4}$, but they are heavier in the absence of the chemical potential, so we will assume that they do not condense and ignore them in our analysis.
} 


\subsection{Pions and longitudinal vectors}

Let us now turn our attention to the pions and longitudinal vectors. For any non-zero momentum, these particles carry the same quantum numbers under the rotation symmetry preserved by the chemical potential, so it is possible for them to mix as the chemical potential is increased. Both the pions and the longitudinal vector excitations involve the two components

$$
A_{i}^{(1,2)}=i k_{i} A_{T}^{(1,2)}, \quad \text { and } \quad A_{0}^{(1,2)} .
$$

Again, the equations are diagonal when we choose

$$
\delta A_{i}^{(1)}= \pm i \delta A_{i}^{(2)}, \quad \delta A_{0}^{(1)}= \pm i \delta A_{0}^{(2)} .
$$

We will use the polarization corresponding to the plus signs below, and keep $\delta A_{0}^{(2)}$ and $\delta A_{i}^{(1)}$ as the two independent components (with the others given by (4.13)); as discussed above, the other polarization gives identical results.

The boundary conditions determine how the pions and longitudinal vectors are encoded in these fields. The general solution to the equations of motion of the two functions $A_{T}$ and $A_{0}$ near the boundary takes the form

$$
\delta A_{T}^{(1)}=c_{0}+\frac{c_{1}}{z}+\cdots, \quad \delta A_{0}^{(2)}=d_{0}+\frac{d_{1}}{z}+\cdots .
$$

Here $c_{0}$ and $d_{0}$ are the coefficients of the non-normalizable modes, while $c_{1}$ and $d_{1}$ are the coefficients of the normalizable modes. The equation of motion (4.8) imposes one constraint on these four free parameters, thereby leaving a two-parameter family after the overall normalization is fixed.

The solutions for the pions are expected to have a non-zero value of $\delta A_{T}$ at $z \rightarrow \pm \infty$ (as for vanishing chemical potential in the $A_{u}=0$ gauge), and we normalize this value to $\pi / 2$. On the other hand, the gauge field $F_{0 i}$ must vanish as $z \rightarrow \pm \infty$, for the solution to have finite action. This constraint determines $d_{0}$ in terms of $c_{0}$, leaving

$$
\text { pion : }\left\{\begin{array}{l}
\delta A_{T}^{(1)}(z \rightarrow \infty)=\frac{\pi}{2}+\frac{c_{1}}{z}+\ldots \\
\delta A_{0}^{(2)}(z \rightarrow \infty)=\left(\omega+\mu_{I}\right) \frac{\pi}{2}+\left(\frac{c_{1} k^{2}}{\omega+\mu_{I}}-\mu_{I}\right) \frac{1}{z}+\ldots
\end{array}\right.
$$

Note that as $\mu_{I} \rightarrow 0$ we have $\delta A_{0}^{(2)}=\omega \delta A_{T}^{(1)}$, as expected due to the enhanced Lorentz symmetry there.

On the other hand, the solutions corresponding to longitudinal vector mesons are characterized by the absence of non-normalizable components in $A_{i}$ and $A_{0}$, so that we have (in an arbitrary normalization)

$$
\text { longitudinal vectors : }\left\{\begin{array}{l}
\delta A_{T}^{(1)}(z \rightarrow \infty)=\frac{1}{z}+\ldots, \\
\delta A_{0}^{(2)}(z \rightarrow \infty)=\frac{k^{2}}{\omega+\mu_{I}} \frac{1}{z}+\ldots
\end{array}\right.
$$




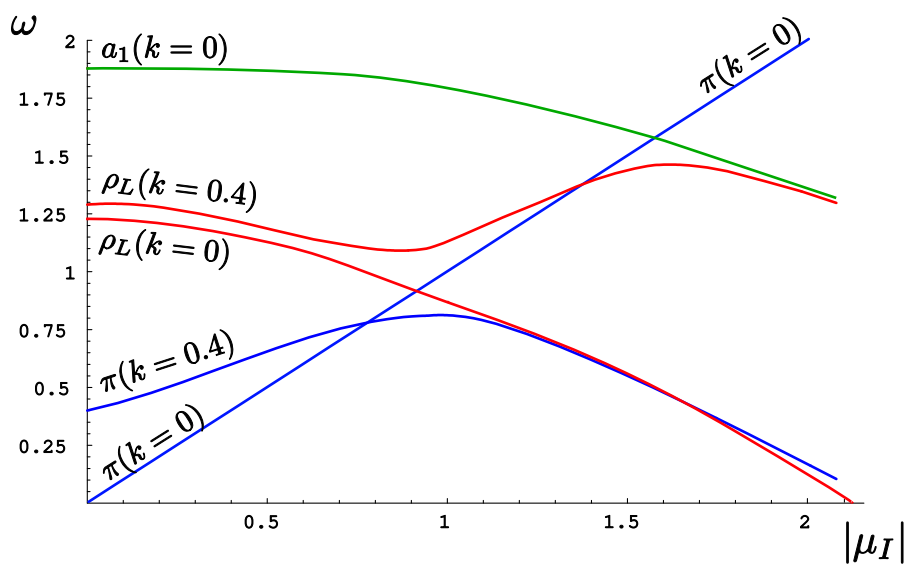

Figure 4: The energy $\omega$ as a function of the isospin chemical potential $\mu_{I}$, in units of $\sqrt{u_{\Lambda} / R_{D 4}^{3}}=2 /(3 R)$, for the sector consisting (at $\mu_{I}=0$ ) of the pion $\pi$ and the first two longitudinal vector mesons $\rho_{L}$ and $a_{1}$. At $\mu_{I} \approx 2.1 * 2 /(3 R)$ the first longitudinal vector meson becomes unstable. This coincides with the instability of the transverse vector meson depicted in figure 3 .

Note that if $c_{1} \rightarrow \infty$ in (4.15), the pion modes look like longitudinal vector modes (with infinite overall normalization); this will be important for us later.

Let us now discuss the relation between the boundary conditions at $z= \pm \infty$. At $\mu_{I}=0$ the pion is encoded in $A_{T}^{(1)}$ which is anti-symmetric in $z$; the relation of the pion to a chiral symmetry transformation implies that this should remain true near the boundary also for non-zero $\mu_{I}$,

$$
A_{T}^{(1)}(z=+\infty)=-A_{T}^{(1)}(z=-\infty) .
$$

For the zeroth component, the boundary condition comes from requiring $F_{0 i}=0$ at the boundary, which gives

$$
\begin{aligned}
& A_{0}^{(2)}(z=+\infty)=\left(\omega+\mu_{I}\right) A_{T}^{(1)}(z=+\infty), \\
& A_{0}^{(2)}(z=-\infty)=\left(\omega-\mu_{I}\right) A_{T}^{(1)}(z=-\infty)=\left(-\omega+\mu_{I}\right) A_{T}^{(1)}(z=+\infty) .
\end{aligned}
$$

Before we discuss the states at generic values of the momentum $k$, let us first analyze the $k=0$ case. This case can in fact be solved analytically, again specializing to the case of $L=\pi R$, where $\bar{A}(z)=2 \arctan \left(z / u_{\Lambda}\right) / \pi$. The equation of motion (4.8) becomes

$$
-2 \mu_{I} \delta A_{0}^{(2)}+u_{\Lambda}\left(1+z^{2} / u_{\Lambda}^{2}\right)\left(\left(\pi \omega+2 \mu_{I} \arctan \left(z / u_{\Lambda}\right)\right) \partial_{z} \delta A_{0}^{(2)}(z)\right)=0 .
$$

This is solved by

$$
\delta A_{0}^{(2)}=\left(\omega+\frac{2 \mu_{I}}{\pi} \arctan \left(z / u_{\Lambda}\right)\right) \cdot \text { const. }
$$

In order to make this satisfy the boundary conditions (4.18) we are forced to choose $\omega=\mu_{I}$. This simple $k=0$ state becomes the pion in the limit $\omega, \mu_{I} \rightarrow 0$. We see that its frequency goes up with $\left|\mu_{I}\right|$ and never vanishes. 
In order to analyze the fate of the remainder of the spectrum in the pion/longitudinal vector sector, we resort to a numerical solution of the equations of motion. Modes satisfying (4.16) are found in the usual way with a one-parameter shooting method, starting from $z=\infty$ and looking for values of $\omega$ such that the solution goes to zero as $z \rightarrow-\infty$. For the modes which are pions at $\mu_{I}=0$, i.e. modes satisfying (4.15), a search through the two-dimensional space spanned by $c_{1}$ and $\omega$ is required. Some details about this procedure can be found in the appendix.

The upshot of this analysis is the following. The modes which start out as longitudinal vectors when $\mu_{I}=0$ all have decreasing $\omega$ as $\mu_{I}$ is increased, and the frequency of the lowest one goes to zero at $\mu_{I}=\mu_{\text {crit }}$. For the modes starting out with pion boundary conditions, the situation is more subtle. They start by going up, but they mix with the other modes. The low-momentum modes starting out as pions develop a large value for $c_{1}$, which goes to infinity as $\mu_{I} \rightarrow \mu_{\text {crit }}$. These modes therefore go over from pure pions to pure longitudinal vectors ${ }^{17}$ as $\mu_{I}$ goes from zero to $\mu_{\text {crit }}$. Similarly, the longitudinal vectors develop a pion component, and as $\mu_{I} \rightarrow \mu_{\text {crit }}$ one linear combination of the modes which starts out as longitudinal vectors may be identified with the pion.

In figure 4 we plot the value of $\omega$ for the pion and longitudinal vector states for a range of values of $\mu_{I}$, at fixed $k$. This plot first of all shows that a small chemical potential leads to massive pions with $\omega^{2}=k^{2}+\mu_{I}^{2}$, in accordance with our analysis of the chiral Lagrangian in the previous section. As $\mu_{I}$ is increased, the frequencies of the zero-momentum pions and of the lightest longitudinal vectors cross each other, and the lightest state becomes a longitudinal vector rather than a pion. Note that for non-zero $k$ the two states cannot cross each other since they then have the same quantum numbers, but instead they mix as described above, and they come closer and closer to level-crossing as $k \rightarrow 0$.

For a value of $\mu_{I} \approx 2.1 * 2 /(3 R)$, the lowest-mass longitudinal vector meson (which we call the "rho") goes to zero frequency (at zero spatial momentum), indicating a potential instability. This is precisely the point where we also found that the lowest-mass transverse vector meson becomes unstable. Thus, the conclusion is that an instability of the rho meson develops at $\mu_{\text {crit }} \approx 2.1 * 2 /(3 R) \approx 1.7 m_{\rho}$, where $m_{\rho}$ is the mass of the rho meson at zero chemical potential. The fact that the transverse vectors and longitudinal vectors go to zero frequency at the same value of $\mu_{I}$ is not surprising, since for zero spatial momentum (where we find the first instability) there is no distinction between transverse and longitudinal vectors.

Note that the Skyrme model, which (as mentioned above) is a good approximation to the low-energy physics of the Sakai-Sugimoto model, indicates that the first instability (the first mode to go to zero frequency) as we increase $\mu_{I}$ comes from pions with $\mu_{I}$ and $k$ of the order of the scale of the Skyrme term (which is of the same order as the rho meson mass in the Sakai-Sugimoto model). This differs from our conclusion that the first instability comes from vector mesons of zero momentum. Of course, this is not a contradiction, since the Skyrme approximation breaks down when $\mu_{I}$ and/or $k$ become of order the mass of

\footnotetext{
${ }^{17}$ Recall that to study the pions we arbitrarily fixed $c_{0}=\pi / 2$, but a more appropriate normalization when $c_{1} \rightarrow \infty$ is $c_{0} \rightarrow 0$ with $c_{1}$ remaining finite.
} 
the vector mesons, and the additional vector fields must be included in the analysis then (as we did in our analysis).

\subsection{Vector meson condensation}

We have seen that at $\mu_{I} \approx 1.7 m_{\rho}$, the zero momentum modes of the "rho" vector meson go to zero frequency. This suggests that the theory becomes unstable there towards forming a condensate of rho mesons, in addition to the pion condensate which is already present; this was conjectured to happen also in $\mathrm{QCD}[5,6,7]$. This condensate is of a vector field, so it breaks the rotational symmetry $\mathrm{SO}(3) \rightarrow \mathrm{SO}(2)$, and since it involves the $\sigma_{1}$ and/or $\sigma_{2}$ components of the meson, it also completely breaks the remaining $U(1)$ flavor symmetry. ${ }^{18}$

In order to find this new ground state, we assume without loss of generality that the third component of the vector rho meson condenses, and that the condensate is proportional to $\sigma_{1}$. We also assume a static and translationally-invariant solution. In this convention, the Goldstone bosons for the broken rotational symmetry are the fluctuations of $A_{2}^{(1)}$ and $A_{1}^{(1)}$, while the fluctuations of $A_{3}^{(2)}$ include the Goldstone boson for the broken flavor $U(1)$. We then have to solve the non-linear equations of motion

$$
\begin{aligned}
& R_{D 4}^{-3} \partial_{u}\left[u^{5 / 2} \gamma^{-1 / 2} \partial_{u} A_{0}^{(3)}\right]=4\left(A_{3}^{(1)}\right)^{2} A_{0}^{(3)} u^{-1 / 2} \gamma^{1 / 2} \\
& R_{D 4}^{-3} \partial_{u}\left[u^{5 / 2} \gamma^{-1 / 2} \partial_{u} A_{3}^{(1)}\right]=-4\left(A_{0}^{(3)}\right)^{2} A_{3}^{(1)} u^{-1 / 2} \gamma^{1 / 2},
\end{aligned}
$$

with the boundary conditions $A_{0}^{(3)}(z= \pm \infty)= \pm \mu_{I} / 2, A_{3}^{(1)}(z= \pm \infty)=0$. We expect that for $\mu_{I}<\mu_{\text {crit }}$ these equations admit only the solution of the previous section which describes the pion condensate, i.e.

$$
\mu_{I}<\mu_{\text {crit }}: \quad A_{0}^{(3)}=\frac{\mu_{I}}{\pi} \arctan \left(\frac{z}{u_{\Lambda}}\right), \quad A_{3}^{(1)}=0 .
$$

For $\mu_{I}>\mu_{\text {crit }}$ we expect the appearance of an additional solution, which has both $A_{0}^{(3)}$ and $A_{3}^{(1)}$ non-zero.

We again resort to a numerical analysis in order to find the solution. Details of this procedure can be found in the appendix. The upshot is that indeed a rho meson condensate forms for $\mu_{I}>\mu_{\text {crit }}$, so there is a second order phase transition there. Around $\mu_{I}=\mu_{\text {crit }}$ this condensate is, to good approximation, given by

$$
\langle\rho\rangle \propto \begin{cases}0 & \text { for } \mu_{I}<\mu_{\text {crit }}, \\ \sqrt{\mu_{I}-\mu_{\text {crit }}} & \text { for } \mu_{I} \geq \mu_{\text {crit }} .\end{cases}
$$

This condensate is drawn in figure 5, using an arbitrary normalization in which we read it off from the coefficient of the normalizable mode of $A_{3}^{(1)}$; it should be possible to translate

\footnotetext{
${ }^{18}$ There is another possible condensate of the vector meson, in which the unbroken $U(1)$ is a diagonal subgroup of the $\mathrm{U}(1)$ flavor symmetry and an $\mathrm{SO}(2)$ rotational symmetry [7]. Without loss of generality, we can assume that in this phase the non-zero components of the gauge field are $A_{1}^{(1)}=A_{2}^{(2)}$ (in addition to $\left.A_{0}^{(3)}\right)$. We have verified numerically that in our model this alternative condensate always has higher energy than the condensate discussed below, so we will not consider it further.
} 


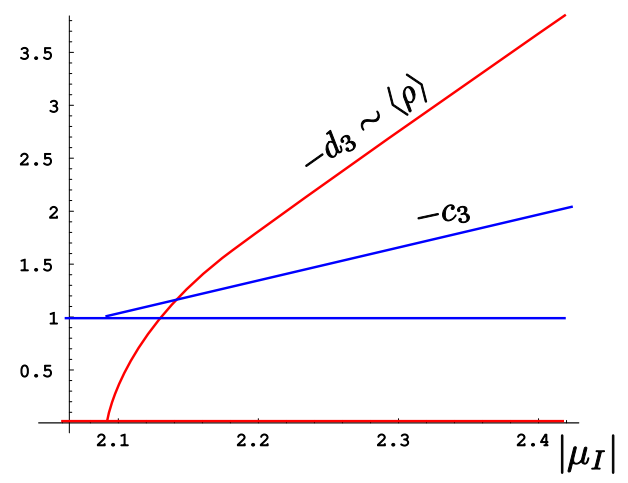

Figure 5: Appearance of the new ground state in which the $\rho$ meson has condensed. This new ground state also still has a non-zero pion condensate. We plot $\left(-c_{3}\right)$ (in units of $u_{\Lambda}$ ) and $\left(-d_{3}\right)$ (in units of $\left(u_{\Lambda} / R_{D_{4}}\right)^{3 / 2}$ ) for both solutions, where $c_{3}$ and $d_{3}$ are defined by $A_{0}^{(3)}=\mu_{I}\left(1 / 2+c_{3} / \pi z+\cdots\right), A_{3}^{(1)}=d_{3} / z+\cdots$.

this to a physical normalization where the meson has a canonical kinetic term, but we have not done this here, and we do not expect this to make a big difference near the phase transition. The value of $\left(-\mu_{I} * c_{3}\right)$ in this figure is the isospin charge density (up to a constant); note that in the solution with the rho condensate it rises more rapidly than in the solution that has only the pion condensate.

The fact that the expectation value of the rho meson scales as $\sqrt{\mu_{I}-\mu_{\text {crit }}}$ near the transition is expected on general grounds, by a Landau-Ginzburg type analysis of the rho meson effective theory, assuming that the effective mass squared of the rho mesons near the transition is proportional to $\left(\mu_{\text {crit }}-\mu_{I}\right)$, and that there is an effective potential of fourth order in the rho mesons which stabilizes the condensate. Our analysis implies that the coefficient of this fourth order term is positive.

\subsection{Potential generalizations}

As indicated above, our stability analysis so far is limited to the special case of $L=\pi R$, and it would be interesting to generalize it to other values of $L / R$, and to see whether the instability of the isotropic phase persists for all values of $L / R$, and how $\mu_{\text {crit }}$ depends on this parameter. For small enough values of $L / R$ there is also an intermediate temperature phase in which the branes are connected [24], and it would be interesting to see if a similar instability arises in that phase as well or not. In that phase there are also deconfined quarks in the spectrum, which may play an interesting role.

Our analysis so far is limited to the first instability which arises as we increase $\mu_{I}$, which leads to a condensation of the lowest vector meson. It would be interesting to see what happens as $\mu_{I}$ is increased further - do additional fields also want to condense? This analysis is more difficult than the analysis we performed here since the solutions for $\mu_{I}>\mu_{\text {crit }}$ which one has to expand around are only known numerically, but still it should be possible to do it. Of course, eventually, as we increase $\mu_{I}$ to order $\lambda_{5} / L^{2}$, we also need to include the DBI and Chern-Simons terms in the action. Baryons may also eventually become relevant for the analysis (recall that in the Sakai-Sugimoto model, the ratio of 
the baryon mass to its isospin scales as $\lambda_{5} / R$ compared to that of the mesons [10,21], so baryons are not expected to be relevant for small $\mu_{I}$ ). It would also be interesting to look for possible non-translationally-invariant solutions.

Naively, our analysis in this section implies that the large $\mu_{I}$ solutions of $\S 3.4$ are never relevant, since the isotropic solutions found in that section do not dominate already for smaller values of $\mu_{I}$. However, there are several situations where these solutions may still be relevant. One is the case of an axial $U(1)$ chemical potential (which is also part of the full phase diagram of QCD, and may be interesting in particular at large $N_{c}$ where the axial symmetry is approximately conserved). In this case there are no instabilities for small values of $\mu$ (for which the spectrum is unmodified), but the Chern-Simons term may well lead to instabilities at larger values of $\mu$ (of order $\lambda_{5} / L^{2}$ ), similar to those discussed in [41]. To see this one has to expand the action of a single D8-brane around the solutions of $\$ 3.4$, using the full DBI plus Chern-Simons action. Another case where these solutions could be relevant is the case of $N_{f}>2$ with generic $S U\left(N_{f}\right)$ isospin chemical potentials, with all eigenvalues different. When $L<\pi R$ this leads to a separation of the D8-branes, which

gives a mass to the off-diagonal matrix elements, and this may eliminate the instabilities discussed in this section which come from off-diagonal elements of the meson field. Again, new instabilities may then arise for $\mu \sim \lambda_{5} / L^{2}$; it would be interesting to investigate this further.

\section{Acknowledgements}

We thank Philippe de Forcrand, Simon Hands, Andreas Karch, David Mateos, Owe Philipsen, Francesco Sannino, Thomas Schäfer, Dam T. Son, Kim Splittorff, Misha Stephanov, Arkady Vainshtein and Larry Yaffe for discussions, and the organizers of the Newton Institute programme "Strong Fields, Integrability and Strings" and the workshop on "Exploring QCD: Deconfinement, Extreme Environments and Holography" for hospitality and for creating a stimulating environment. OA would also like to thank Stanford University, SLAC and Tel-Aviv University for hospitality during the course of this project.

The work of OA and JS is supported in part by a center of excellence supported by the Israel Science Foundation (grant number 1468/06), by a grant (DIP H52) of the German Israel Project Cooperation, and by the European Network MRTN-CT-2004-512194. The work of OA is also supported in part by the Israel-U.S. Binational Science Foundation and by a grant from the G.I.F., the German-Israeli Foundation for Scientific Research and Development. The work of OA at the Newton Institute was supported by a Senior Visiting Fellowship funded by EPSRC grant 531174. The work of KP was supported by VIDI grant 016.069.313 from the Dutch Organization for Scientific Research (NWO). 


\section{Appendix: physical modes}

In this appendix we provide some more technical details related to the determination of the meson spectrum, as well as the construction of the new ground state in which the rho meson is condensed. This analysis is, in both cases, essentially based on a standard shooting method for the two-point boundary value problem. However, there are a few interesting features which we would like to highlight.

Let us first discuss the construction of solutions with asymptotic expansion (4.15) and boundary conditions (4.18). When solving for the values of $c_{1}$ and $\omega$ for which such a mode exists, by shooting from $z=\infty$ to $z=-\infty$, it is instructive to plot two curves, one for which the $A_{0}^{(2)}$ boundary condition is satisfied at $z=-\infty$ and one for which the $A_{T}^{(1)}$ boundary condition at $z=-\infty$ holds true. This leads to figure 6. The intersection points of these two types of curves indicate physical states. The intersection which is almost on the $\omega$-axis has a $1 / z$ coefficient (in $A_{T}^{(1)}$ ) which is of similar magnitude as the asymptotic value of $A_{T}^{(1)}$ (and thus corresponds to the pion). The other intersection point, away from the $\omega$-axis, is a longitudinal vector (when the $1 / z$ coefficient of these modes is normalized to one, their value at $z= \pm \infty$ goes to zero as $k \rightarrow 0)$. Following the figures, there is a clear sense in which a state which starts out as a pion eventually turns into a longitudinal vector, and vice versa, as $\mu_{I}$ is increased. As discussed in $\S 4.3$, this transition is smooth for any non-zero momentum.
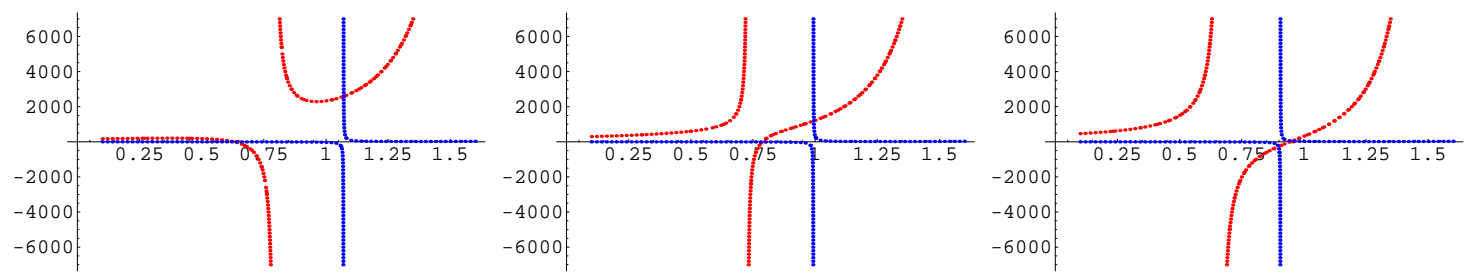

$\mu_{I}=0.63$

$\mu_{I}=0.79$

$\mu_{I}=0.94$

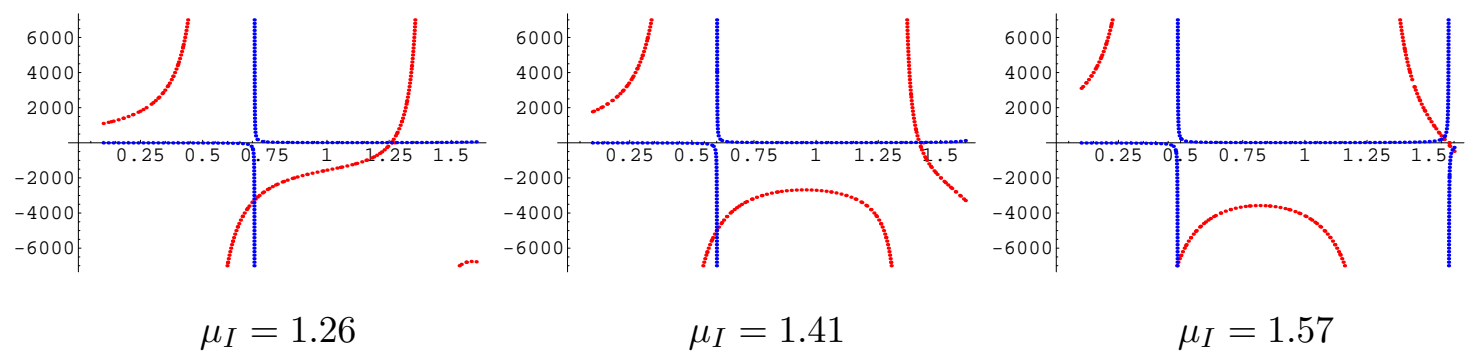

Figure 6: The location of the physical modes for small and fixed $k\left(k=0.05 \sqrt{u_{\Lambda} / R_{D 4}^{3}}\right)$ for various values of $\mu_{I}$ (in units of $\sqrt{u_{\Lambda} / R_{D 4}^{3}}=2 /(3 R)$ ). The horizontal and vertical axes label $\omega$ (in the same units) and the coefficient of the $1 / z$ term in $A_{T}^{(1)}$ (in units of $u_{\Lambda}$, in the normalization of (4.15), respectively. The blue, almost straight curves indicate where the boundary condition on $A_{T}^{(1)}(z=-\infty)$ is satisfied, while the red curves do the same for $A_{0}^{(2)}$. 


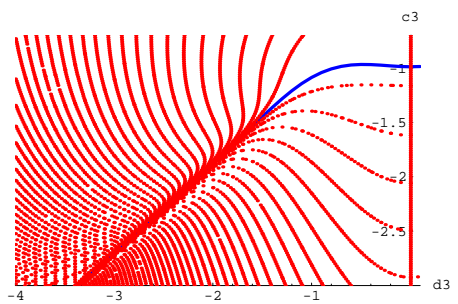

$\mu_{I}=1.57$

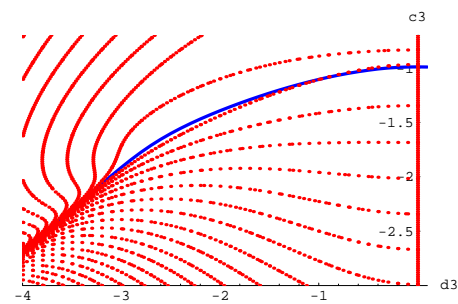

$\mu_{I}=2.10$

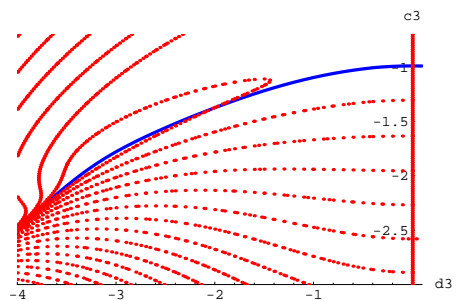

$\mu_{I}=2.20$

Figure 7: The location of solutions to the non-linear equations of motion (4.21) for the rho meson condensate, including the new ground state, in the same units as in figure 5 . Blue curves indicate the parameter values for which $A_{0}^{(3)}(z=-\infty)$ satisfies the boundary condition, while red dots do the same for $A_{3}^{(1)}(z=-\infty)$.

For the construction of the rho meson condensate, a scan is required through the space of $c_{3}$ and $d_{3}$ coefficients in the expansion

$$
A_{0}^{(3)}=\mu_{I}\left(\frac{1}{2}+\frac{c_{3}}{\pi z}\right)+\ldots, \quad A_{3}^{(1)}=\frac{d_{3}}{z}+\ldots .
$$

Again we plot the curves at which $A_{0}^{(3)}(z=-\infty)$ and $A_{3}^{(1)}(z=-\infty)$ satisfy the boundary conditions, and look for intersection points. The pion condensate solution $c_{3}=-1, d_{3}=0$ satisfies (4.21) for all values of $\mu_{I}$; this is the point on the vertical axis in figure 7 . For $\mu_{I}>\mu_{\text {crit }}$ a new intersection point develops.

\section{References}

[1] T. Schäfer, "Phases of QCD", hep-ph/0509068.

[2] M. G. Alford, A. Kapustin, and F. Wilczek, "Imaginary chemical potential and finite fermion density on the lattice", Phys. Rev. D59 (1999) 054502, hep-lat/9807039.

[3] D. T. Son and M. A. Stephanov, "QCD at finite isospin density", Phys. Rev. Lett. 86 (2001) 592-595, hep-ph/0005225.

[4] D. T. Son and M. A. Stephanov, "QCD at finite isospin density: From pion to quark antiquark condensation", Phys. Atom. Nucl. 64 (2001) 834-842, hep-ph/0011365.

[5] D. N. Voskresensky, "On the possibility of the condensation of the charged rho meson field in dense isospin asymmetric baryon matter", Phys. Lett. B392 (1997) 262.

[6] J. T. Lenaghan, F. Sannino, and K. Splittorff, "The superfluid and conformal phase transitions of two-color QCD", Phys. Rev. D65 (2002) 054002, hep-ph/0107099.

[7] F. Sannino, "General structure of relativistic vector condensation", Phys. Rev. D67 (2003) 054006, hep-ph/0211367.

[8] F. Sannino, "Review of vector condensation at high chemical potential", AIP Conf. Proc. 688 (2004) 121-127, hep-ph/0307053.

[9] J. B. Kogut and D. K. Sinclair, "Lattice QCD at finite isospin density at zero and finite temperature", Phys. Rev. D66 (2002) 034505, hep-lat/0202028. 
[10] T. Sakai and S. Sugimoto, "Low energy hadron physics in holographic QCD", Prog. Theor. Phys. 113 (2005) 843-882, hep-th/0412141.

[11] T. Sakai and S. Sugimoto, "More on a holographic dual of QCD", Prog. Theor. Phys. 114 (2006) 1083-1118, hep-th/0507073.

[12] K. Peeters and M. Zamaklar, "The string/gauge theory correspondence in QCD", Lectures presented at the 45th Schladming Winter School on Theoretical Physics, arXiv:0708.1502 [hep-ph].

[13] T. Albash, V. G. Filev, C. V. Johnson and A. Kundu, "Global currents, phase transitions, and chiral symmetry breaking in large $N_{c}$ gauge theory," hep-th/0605175.

S. Nakamura, Y. Seo, S.-J. Sin, and K. P. Yogendran, "A new phase at finite quark density from AdS/CFT", hep-th/0611021.

S. Kobayashi, D. Mateos, S. Matsuura, R. C. Myers, and R. M. Thomson, "Holographic phase transitions at finite baryon density", JHEP 02 (2007) 016, hep-th/0611099.

J. Erdmenger, M. Kaminski, and F. Rust, "Isospin diffusion in thermal AdS/CFT with flavor", Phys. Rev. D76 (2007) 046001, arXiv:0704.1290 [hep-th].

S.-J. Sin, "Gravity back-reaction to the baryon density for bulk filling branes", arXiv:0707.2719 [hep-th].

S. Nakamura, Y. Seo, S.-J. Sin, and K. P. Yogendran, "Baryon-charge chemical potential in AdS/CFT", arXiv:0708.2818 [hep-th].

K. Ghoroku, M. Ishihara, and A. Nakamura, "D3/D7 holographic gauge theory and chemical potential", arXiv:0708.3706 [hep-th].

A. Karch and A. O'Bannon, "Holographic thermodynamics at finite baryon density: Some exact results", arXiv:0709.0570 [hep-th].

D. Mateos, S. Matsuura, R. C. Myers, and R. M. Thomson, "Holographic phase transitions at finite chemical potential", arXiv:0709.1225 [hep-th].

Y. Kim, C.-H. Lee, and H.-U. Yee, "Holographic nuclear matter in AdS/QCD", arxiv:0707.2637 [hep-ph].

K.-I. Kim, Y. Kim, and S. H. Lee, "Isospin matter in AdS/QCD", arXiv:0709.1772 [hep-ph].

[14] O. Bergman, G. Lifschytz, and M. Lippert, "Holographic nuclear physics", arXiv:0708.0326 [hep-th].

[15] M. Rozali, H.-H. Shieh, M. Van Raamsdonk, and J. Wu, "Cold Nuclear Matter In Holographic QCD", arXiv:0708.1322 [hep-th].

[16] D. Yamada, "Sakai-Sugimoto model at high density", arXiv:0707.0101 [hep-th].

[17] J. L. Davis, M. Gutperle, P. Kraus, and I. Sachs, "Stringy NJL and Gross-Neveu models at finite density and temperature", arXiv:0708.0589 [hep-th].

[18] K.-Y. Kim, S.-J. Sin, and I. Zahed, "The chiral model of Sakai-Sugimoto at finite baryon density", arXiv:0708.1469 [hep-th].

[19] E. Shuster and D. T. Son, "On finite-density QCD at large $N_{c}$ ", Nucl. Phys. B573 (2000) 434-446. 
[20] K. Nawa and H. Suganuma, and T. Kojo, "Baryons in holographic QCD", Phys. Rev. D75 (2007) 086003, hep-th/0612187.

[21] H. Hata, T. Sakai, S. Sugimoto, and S. Yamato, "Baryons from instantons in holographic QCD", hep-th/0701280.

[22] D. K. Hong, M. Rho, H.-U. Yee, and P. Yi, "Chiral dynamics of baryons from string theory", hep-th/0701276.

[23] D. K. Hong, M. Rho, H.-U. Yee, and P. Yi, "Dynamics of baryons from string theory and vector dominance", arXiv:0705.2632 [hep-th].

[24] O. Aharony, J. Sonnenschein, and S. Yankielowicz, "A holographic model of deconfinement and chiral symmetry restoration", Ann. Phys. 322 (2007) 1420-1443, hep-th/0604161.

[25] A. Parnachev and D. A. Sahakyan, "Chiral phase transition from string theory", hep-th/0604173.

[26] M. Kruczenski, L. A. P. Zayas, J. Sonnenschein, and D. Vaman, "Regge trajectories for mesons in the holographic dual of large- $N_{c}$ QCD", JHEP 06 (2005) 046, hep-th/0410035.

[27] K. Peeters, J. Sonnenschein, and M. Zamaklar, "Holographic decays of large-spin mesons", JHEP 02 (2006) 009, hep-th/0511044.

[28] O. Bergman, S. Seki, and J. Sonnenschein, "Quark mass and condensate in HQCD", arXiv:0708.2839 [hep-th].

[29] A. Dhar and P. Nag, "Sakai-Sugimoto model, tachyon condensation and chiral symmetry breaking", arXiv:0708.3233 [hep-th].

[30] R. Casero, E. Kiritsis, and A. Paredes, "Chiral symmetry breaking as open string tachyon condensation", hep-th/0702155.

[31] K. Peeters, J. Sonnenschein, and M. Zamaklar, "Holographic melting and related properties of mesons in a quark gluon plasma", Phys. Rev. D74 (2006) 106008, hep-th/0606195.

[32] N. Horigome and Y. Tanii, "Holographic chiral phase transition with chemical potential", JHEP 01 (2007) 072, hep-th/0608198.

[33] A. Parnachev and D. A. Sahakyan, "Photoemission with chemical potential from QCD gravity dual", Nucl. Phys. B768 (2007) 177-192, hep-th/0610247.

[34] A. Parnachev, "Holographic QCD with isospin chemical potential", arXiv:0708.3170 [hep-th].

[35] E. Witten, "Anti-de Sitter space, thermal phase transition, and confinement in gauge theories", Adv. Theor. Math. Phys. 2 (1998) 505, hep-th/9803131.

[36] J. M. Maldacena, "The large- $N$ limit of superconformal field theories and supergravity", Adv. Theor. Math. Phys. 2 (1998) 231-252, hep-th/9711200.

[37] E. Witten, "Current Algebra Theorems for the $U(1)$ Goldstone Boson", Nucl. Phys. B156 (1979) 269.

[38] G. Veneziano, "U(1) Without Instantons", Nucl. Phys. B159 (1979) 213-224.

[39] O. Bergman and G. Lifschytz, "Holographic $U(1)_{A}$ and string creation", JHEP 04 
(2007) 043, hep-th/0612289.

[40] K. Splittorff, D. T. Son, and M. A. Stephanov, "QCD-like theories at finite baryon and isospin density", Phys. Rev. D64 (2001) 016003, hep-ph/0012274.

[41] S. K. Domokos and J. A. Harvey, "Baryon number-induced Chern-Simons couplings of vector and axial-vector mesons in holographic QCD", arXiv:0704.1604 [hep-ph]. 